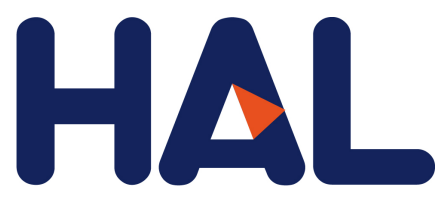

archives-ouvertes

\title{
Fermi-LAT Observations of High-energy Behind-the-limb Solar Flares
}

M. Ackermann, A. Allafort, L. Baldini, G. Barbiellini, D. Bastieri, R. Bellazzini, E. Bissaldi, R. Bonino, E. Bottacini, J. Bregeon, et al.

\section{- To cite this version:}

M. Ackermann, A. Allafort, L. Baldini, G. Barbiellini, D. Bastieri, et al.. Fermi-LAT Observations of High-energy Behind-the-limb Solar Flares. Astrophys.J., 2017, 835 (2), pp.219. 10.3847/1538$4357 / 835 / 2 / 219$. hal-01554386

\section{HAL Id: hal-01554386 \\ https://hal.archives-ouvertes.fr/hal-01554386}

Submitted on 3 Jun 2020

HAL is a multi-disciplinary open access archive for the deposit and dissemination of scientific research documents, whether they are published or not. The documents may come from teaching and research institutions in France or abroad, or from public or private research centers.
L'archive ouverte pluridisciplinaire HAL, est destinée au dépôt et à la diffusion de documents scientifiques de niveau recherche, publiés ou non, émanant des établissements d'enseignement et de recherche français ou étrangers, des laboratoires publics ou privés. 


\section{Fermi-LAT Observations of High-energy Behind-the-limb Solar Flares}

M. Ackermann ${ }^{1}$, A. Allafort ${ }^{2}$, L. Baldini ${ }^{3}$, G. Barbiellini ${ }^{4,5}$, D. Bastieri ${ }^{6,7}$, R. Bellazzini ${ }^{8}$, E. Bissaldi ${ }^{9}$, R. Bonino ${ }^{10,11}$, E. Bottacini ${ }^{2}$, J. Bregeon ${ }^{12}$, P. Bruel ${ }^{13}$, R. Buehler ${ }^{1}$, R. A. Cameron ${ }^{2}$, M. Caragiulo ${ }^{9,14}$, P. A. Caraveo ${ }^{15}$, E. Cavazzuti ${ }^{16}$, C. Cechi ${ }^{17,18}$, E. Charles ${ }^{2}$, S. Ciprini ${ }^{16,17}$, F. Costanza ${ }^{9}$, S. Cutini ${ }^{16,17}$, F. D’Ammando ${ }^{19,20}$, F. de Palma ${ }^{9,21}$, R. Desiante ${ }^{10,22}$, S. W. Digel ${ }^{2}$, N. Di Lalla ${ }^{3}$, M. Di Mauro ${ }^{2}$, L. Di Venere, ${ }^{9,14}$, P. S. Drell ${ }^{2}$, C. Favuzzi ${ }^{9,14}$, Y. Fukazawa ${ }^{23}$, P. Fusco 9,14 , F. Gargano ${ }^{9}$, N. Giglietto ${ }^{9,14}$, F. Giordano ${ }^{9,14}$, M. Giroletti ${ }^{19}$, I. A. Grenier ${ }^{24}$, L. Guillemot ${ }^{25,26}$, S. Guiriec ${ }^{27,50}$, T. Jogler ${ }^{28}$, G. Jóhannesson ${ }^{29,30}$,

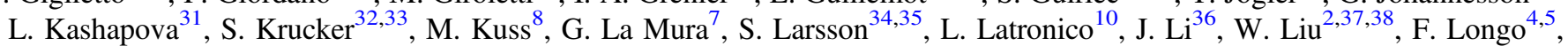
F. Loparco ${ }^{9,14}$, P. Lubrano ${ }^{17}$, J. D. Magill ${ }^{39}$, S. Maldera ${ }^{10}$, A. Manfreda ${ }^{3}$, M. N. Mazziotta ${ }^{9}$, W. Mitthumsiri ${ }^{40}$, T. Mizuno ${ }^{41}$, M. E. Monzani ${ }^{2}$, A. Morsellii ${ }^{42}$, I. V. Moskalenko ${ }^{2}$, M. Negro ${ }^{10,11}$, E. Nuss ${ }^{12}$, T. Ohsugii ${ }^{41}$, N. Omodei ${ }^{2}$, E. Orlando ${ }^{2}$, V. Pal'shin ${ }^{43}$, D. Paneque ${ }^{44}$, J. S. Perkins ${ }^{27}$, M. Pesce-Rollins ${ }^{8}$, V. Petrosian ${ }^{2}$, F. Piron ${ }^{12}$, G. Principe ${ }^{45}$, S. Rainò ${ }^{9,14}$, R. Rando ${ }^{6,7}$, M. Razzano ${ }^{8,51}{ }^{1}$, O. Reimer ${ }^{2,46}$, F. Rubio da Costa $^{2}$, C. Sgrò ${ }^{8}$, D. Simone ${ }^{9}$, E. J. Siskind ${ }^{47}$, F. Spada ${ }^{8}$, G. Spandre ${ }^{8}$, P. Spinelli ${ }^{9,14}$, H. Tajima ${ }^{2,48}$, J. B. Thayer ${ }^{2}$, D. F. Torres ${ }^{36,49}$, E. Troja ${ }^{27,39}$, and G. Vianello ${ }^{2}$

${ }^{1}$ Deutsches Elektronen Synchrotron DESY, D-15738 Zeuthen, Germany

${ }^{2}$ W. W. Hansen Experimental Physics Laboratory, Kavli Institute for Particle Astrophysics and Cosmology, Department of Physics and SLAC National Accelerator Laboratory, Stanford University, Stanford, CA 94305, USA; nicola.omodei@stanford.edu, vahep@ stanford.edu

${ }^{3}$ Università di Pisa and Istituto Nazionale di Fisica Nucleare, Sezione di Pisa I-56127 Pisa, Italy

${ }^{4}$ Istituto Nazionale di Fisica Nucleare, Sezione di Trieste, I-34127 Trieste, Italy

${ }^{5}$ Dipartimento di Fisica, Università di Trieste, I-34127 Trieste, Italy

${ }^{6}$ Istituto Nazionale di Fisica Nucleare, Sezione di Padova, I-35131 Padova, Italy

${ }^{7}$ Dipartimento di Fisica e Astronomia "G. Galilei," Università di Padova, I-35131 Padova, Italy

${ }^{8}$ Istituto Nazionale di Fisica Nucleare, Sezione di Pisa, I-56127 Pisa, Italy; melissa.pesce.rollins@pi.infn.it ${ }_{9}$ Istituto Nazionale di Fisica Nucleare, Sezione di Bari, I-70126 Bari, Italy

${ }^{10}$ Istituto Nazionale di Fisica Nucleare, Sezione di Torino, I-10125 Torino, Italy

${ }^{11}$ Dipartimento di Fisica, Università degli Studi di Torino, I-10125 Torino, Italy

${ }^{12}$ Laboratoire Univers et Particules de Montpellier, Université Montpellier, CNRS/IN2P3, F-34095 Montpellier, France

${ }^{13}$ Laboratoire Leprince-Ringuet, École polytechnique, CNRS/IN2P3, F-91128 Palaiseau, France

${ }^{14}$ Dipartimento di Fisica "M. Merlin" dell’Università e del Politecnico di Bari, I-70126 Bari, Italy

${ }^{15}$ INAF-Istituto di Astrofisica Spaziale e Fisica Cosmica Milano, via E. Bassini 15, I-20133 Milano, Italy

${ }_{16}$ Agenzia Spaziale Italiana (ASI) Science Data Center, I-00133 Roma, Italy

${ }_{17}^{17}$ Istituto Nazionale di Fisica Nucleare, Sezione di Perugia, I-06123 Perugia, Italy

${ }^{18}$ Dipartimento di Fisica, Università degli Studi di Perugia, I-06123 Perugia, Italy

${ }^{19}$ INAF Istituto di Radioastronomia, I-40129 Bologna, Italy

${ }^{20}$ Dipartimento di Astronomia, Università di Bologna, I-40127 Bologna, Italy

${ }^{21}$ Università Telematica Pegaso, Piazza Trieste e Trento, 48, I-80132 Napoli, Italy ${ }^{22}$ Università di Udine, I-33100 Udine, Italy

${ }^{23}$ Department of Physical Sciences, Hiroshima University, Higashi-Hiroshima, Hiroshima 739-8526, Japan

${ }^{24}$ Laboratoire AIM, CEA-IRFU/CNRS/Université Paris Diderot, Service d'Astrophysique, CEA Saclay, F-91191 Gif sur Yvette, France

${ }^{25}$ Laboratoire de Physique et Chimie de l'Environnement et de l'Espace-Université d'Orléans/CNRS, F-45071 Orléans Cedex 02, France

${ }^{26}$ Station de radioastronomie de Nançay, Observatoire de Paris, CNRS/INSU, F-18330 Nançay, France

${ }^{27}$ NASA Goddard Space Flight Center, Greenbelt, MD 20771, USA

${ }^{28}$ Friedrich-Alexander-Universität, Erlangen-Nürnberg, Schlossplatz 4, D-91054 Erlangen, Germany

${ }_{30}^{29}$ Science Institute, University of Iceland, IS-107 Reykjavik, Iceland

${ }^{30}$ NORDITA, Roslagstullsbacken 23, SE-106 91 Stockholm, Sweden

${ }^{31}$ Institute of Solar-Terrestrial SB RAS, Lermontov st. 126a, 6640333, Irkutsk, Russia

${ }^{32}$ University of Applied Sciences and Arts Northwestern Switzerland, CH-5210 Windisch, Switzerland

${ }^{33}$ Space Science Laboratory, University of California, Berkeley, CA 94720-7450, USA
${ }^{34}$ Department of Physics, KTH Royal Institute of Technology, AlbaNova, SE-106 91 Stockholm, Sweden

${ }^{5}$ The Oskar Klein Centre for Cosmoparticle Physics, AlbaNova, SE-106 91 Stockholm, Sweden

${ }^{36}$ Institute of Space Sciences (IEEC-CSIC), Campus UAB, Carrer de Magrans s/n, E-08193 Barcelona, Spain

${ }^{37}$ Bay Area Environmental Research Institute, 625 2nd Street, Suite 209, Petaluma, CA 94952, USA

${ }^{38}$ Lockheed Martin Solar and Astrophysics Laboratory, 3251 Hanover Street, Bldg. 252, Palo Alto, CA 94304, USA

39 Department of Physics and Department of Astronomy, University of Maryland, College Park, MD 20742, USA

${ }^{40}$ Department of Physics, Faculty of Science, Mahidol University, Bangkok 10400, Thailand

${ }^{41}$ Hiroshima Astrophysical Science Center, Hiroshima University, Higashi-Hiroshima, Hiroshima 739-8526, Japan

${ }^{42}$ Istituto Nazionale di Fisica Nucleare, Sezione di Roma "Tor Vergata," I-00133 Roma, Italy

${ }^{43}$ St. Peterburg, Vedeneeva 2-31, Russia

${ }^{44}$ Max-Planck-Institut für Physik, D-80805 München, Germany

${ }^{45}$ Erlangen Centre for Astroparticle Physics, D-91058 Erlangen, Germany

${ }^{46}$ Institut für Astro- und Teilchenphysik and Institut für Theoretische Physik, Leopold-Franzens-Universität Innsbruck, A-6020 Innsbruck, Austria

${ }^{47}$ NYCB Real-Time Computing Inc., Lattingtown, NY 11560-1025, USA

${ }_{48}^{48}$ Solar-Terrestrial Environment Laboratory, Nagoya University, Nagoya 464-8601, Japan

${ }^{49}$ Institució Catalana de Recerca i Estudis Avançats (ICREA), E-08010 Barcelona, Spain

Received 2016 October 13; revised 2016 November 23; accepted 2016 December 31; published 2017 January 31

\footnotetext{
${ }_{51}^{5}$ NASA Postdoctoral Program Fellow, USA.

${ }^{51}$ Funded by contract FIRB-2012-RBFR12PM1F from the Italian Ministry of Education, University and Research (MIUR).
} 


\begin{abstract}
We report on the Fermi-LAT detection of high-energy emission from the behind-the-limb (BTL) solar flares that occurred on 2013 October 11, and 2014 January 6 and September 1. The Fermi-LAT observations are associated with flares from active regions originating behind both the eastern and western limbs, as determined by STEREO. All three flares are associated with very fast coronal mass ejections (CMEs) and strong solar energetic particle events. We present updated localizations of the $>100 \mathrm{MeV}$ photon emission, hard X-ray (HXR) and EUV images, and broadband spectra from $10 \mathrm{keV}$ to $10 \mathrm{GeV}$, as well as microwave spectra. We also provide a comparison of the BTL flares detected by Fermi-LAT with three on-disk flares and present a study of some of the significant quantities of these flares as an attempt to better understand the acceleration mechanisms at work during these occulted flares. We interpret the HXR emission to be due to electron bremsstrahlung from a coronal thin-target loop top with the accelerated electron spectra steepening at semirelativistic energies. The $>100 \mathrm{MeV}$ gamma-rays are best described by a pion-decay model resulting from the interaction of protons (and other ions) in a thick-target photospheric source. The protons are believed to have been accelerated (to energies $>10 \mathrm{GeV}$ ) in the CME environment and precipitate down to the photosphere from the downstream side of the CME shock and landed on the front side of the Sun, away from the original flare site and the HXR emission.
\end{abstract}

Key words: Sun: flares - Sun: X-rays, gamma rays

\section{Introduction}

Gamma-ray emission from solar flares is generally believed to occur predominantly in compact high-density regions near the photospheric footpoints of magnetic field lines. Observations of gamma-ray emission from flares whose host active regions (ARs) are located behind the visible solar disk pose interesting questions regarding the acceleration sites and mechanism, the transport and interaction points of the accelerated particles during these rare events.

Three behind-the-limb (BTL) flares with emission up to $100 \mathrm{MeV}$ were observed during solar cycles 21 and 22. The first occulted solar flare whose AR was estimated to be $15^{\circ}$ behind the western limb was observed on 1989 September 29 by the Gamma-Ray Spectrometer (GRS) on board the Solar Maximum Mission (SMM). Vestrand \& Forrest (1993) reported intense gamma-ray line emission in the $1-8 \mathrm{MeV}$ range and a strong $2.223 \mathrm{MeV}$ neutron capture line from this flare. Given the strength of the line emission, it was concluded that a spatially extended component was required in order to explain the observations. The second, detected by PHEBUS on GRANAT (Barat et al. 1994) on 1991 June 1, had intense gamma-ray line emission in the $1-8 \mathrm{MeV}$ range but no neutron capture line, indicating that the emission was of coronal origin. The third occulted flare, detected by PHEBUS, BATSE, and EGRET on 1991 June 30, was an electron-dominated flare with no detectable line emission but with significant emission up to almost $100 \mathrm{MeV}$. Vilmer et al. (1999) report that the spectral properties of this flare were similar to those of the flares occurring on the visible disk. Although there was some speculation and some scenarios were put forth (Cliver et al. 1993), no definite explanations were found on how and where the particles responsible for these emissions were accelerated and where the gamma-rays were produced.

Hard X-ray (HXR) emissions from the loop top of flares originating from ARs located just beyond the limb is often observed by RHESSI (Saldanha et al. 2008). These are referred to as partially occulted flares since only the emission from the loop footpoints are occulted. An unusual such flare was reported by Krucker et al. (2007), where the flare originated $40^{\circ}$ behind the limb. This is very similar to the 2014 September 1 flare discussed in this paper.

The Fermi-Large Area Telescope (LAT) (Atwood et al. 2009) observations have doubled the number of occulted flares detected and provided the first detections of emission in the $\mathrm{GeV}$ range from such rare events. The Fermi-LAT observations sample flares from ARs originating from behind both the eastern and western limbs; all flares are associated with very fast coronal massive ejections (CMEs) and strong solar energetic particle (SEP) events. In this paper, we present the observations of the first three BTL flares detected by FermiLAT together with those from complementary instruments such as STEREO, RHESSI, Konus-Wind (Aptekar et al. 1995), and Fermi-GBM (GBM; Meegan et al. 2009), as well as solar observatories in the Radio Solar Telescope Network (RSTN).

In Section 2 we present the multiwavelength features including the light curves (LCs) and localization of the various emissions. Then in Section 3 we describe a more detailed analysis of the spectral data and compare the properties of the BTL flares with three on-disk flares observed by Fermi-LAT. In Section 4 we discuss the associated SEP observations, and in Section 5 we present a brief summary and interpretation of these results.

\section{Observations of Fermi-LAT BTL Flares}

We have collected all relevant observations of the three BTL flares detected by Fermi-LAT. In what follows we present these observations. A first analysis of the SOL2013-10-11 flare based on Pass7_REP data was presented in Pesce-Rollins et al. (2015). We have reanalyzed all three of the flares using Pass 8 data. The Pass 8 data benefit ${ }^{52}$ from an improved point-spread function, effective area, and energy reach.

\subsection{Light Curves}

SOL2013-10-11: On 2013 October 11 (Oct13), between 07:05:51 and 07:10:51 UT, STEREO-B detected a solar flare with an AR located at N21E103. GOES detected an M1.5 class flare starting at around the same time as STEREO-B. However, based on the STEREO-B $195 \AA$ emission and the method described in Nitta et al. (2013), we estimate that the GOES class of this flare had the AR not been occulted would have been M4.9 with an uncertainty within a factor of three. This method utilizes the pre-flare background-subtracted, fulldisk integrated EUV intensity, as shown in Figure 1(b). A fast

\footnotetext{
52 A summary of the Fermi-LAT Pass 8 performance can be found at http:// www.slac.stanford.edu/exp/glast/groups/canda/lat_Performance.htm.
} 


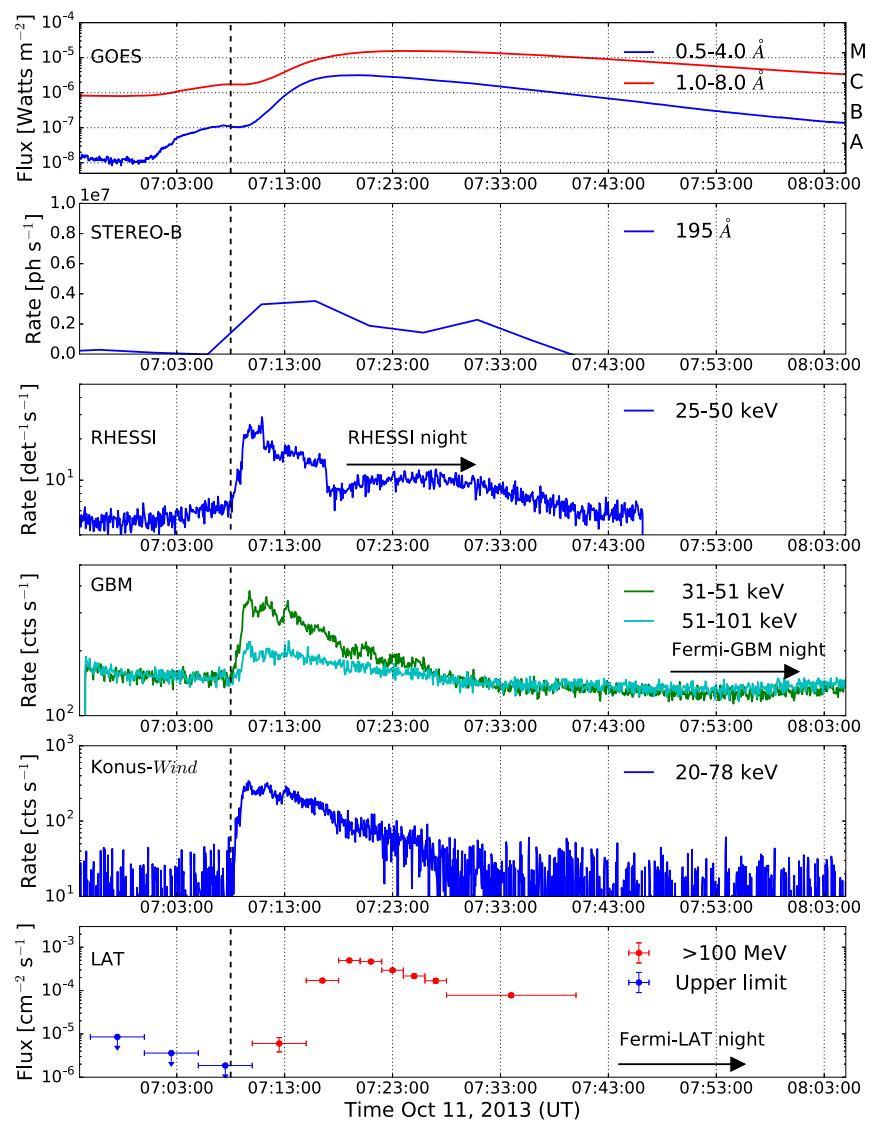

Figure 1. Composite light curve for Oct13 with data from GOES, STEREO, RHESSI, Fermi-GBM, Konus-Wind, and Fermi-LAT. The vertical dashed line represents the estimated start time of the flare, 7:08 UT based on STEREO-B observations. RHESSI night started at around 07:15 UT and Fermi-LAT night started around 07:43 UT.

CME was observed by LASCO with a reported first appearance by the white light coronagraph $\mathrm{C} 2$ (imaging from 2-6 solar radii) at 07:24:10 UT and a linear speed of $1200 \mathrm{~km} \mathrm{~s}^{-1}$. Fermi coverage started at 07:08:00 UT and continued for more than 30 minutes. Fermi-LAT detected $>100 \mathrm{MeV}$ emission for $\sim 30$ minute with the maximum of the flux occurring between 07:20:00-07:25:00 UT. ${ }^{53}$ The Fermi-GBM detection of HXRs began a few minutes before Fermi-LAT and peaked earlier $(\sim 07: 10$ above $50 \mathrm{keV})$. RHESSI coverage was from 07:08:00-07:16:40 UT, overlapping with Fermi for 9 minutes. Konus-Wind, located at Lagrangian point L1, detected emission in the $20-78 \mathrm{keV}$ and $78-310 \mathrm{keV}$ energy bands simultaneously with RHESSI and Fermi-GBM. This flare was also observed in radio by the RSTN and the Nobeyama radio polarimeter (Nakajima et al. 1994) at frequencies up to $9 \mathrm{GHz}$ (see Section 3.4).

The LCs for the GOES, STEREO-B, RHESSI, Fermi-GBM, Konus-Wind, and Fermi-LAT are shown in Figure 1. The microwave (MW) emission is compared with the HXR LC from Konus-Wind in Figure 2.

SOL2014-01-06: On 2014 January 6 (Jan14), between 07:35:46 and 07:45:46 UT, a solar flare erupted from an AR located at S8W110. Both STEREO spacecraft had a full view of the AR and detected a large filament eruption from the AR at

\footnotetext{
$\overline{53}$ From the reanalysis of this flare with Pass 8 data, we gain 5 minutes of additional emission with respect to our previous work (Pesce-Rollins et al. 2015).
}
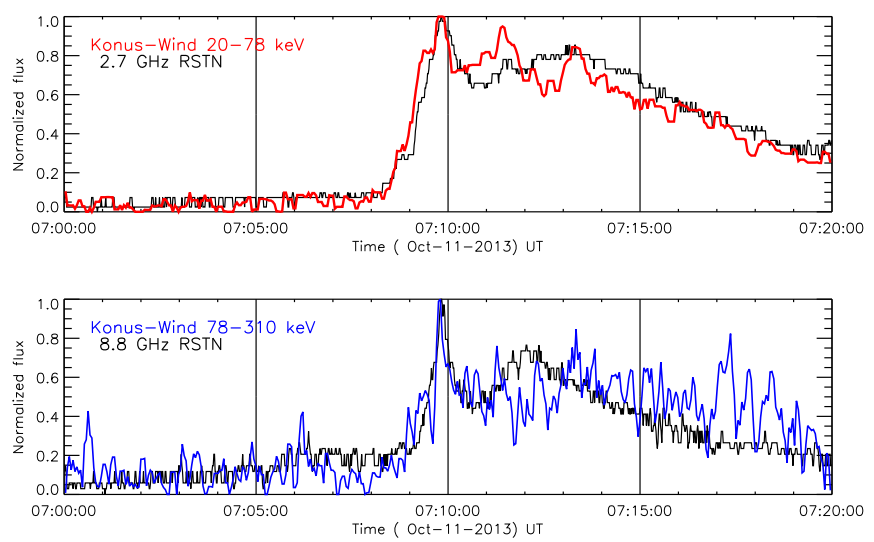

Figure 2. Comparison of the time profiles of HXR and microwave (MW) emission for Oct13. The top and bottom panels compare the 20-78 keV HXR light curve with optically thick gyro-synchrotron (GS) emission at $2.7 \mathrm{GHz}$ and the $78-310 \mathrm{keV}$ HXR light curve with the optically thin GS emission at $8.8 \mathrm{GHz}$, respectively. Note that the shape of the GS spectrum is such that for frequencies below the peak the spectrum is considered optically thick, whereas above the peak is it optically thin.

approximately 07:50:00 UT. The tip of this filament was seen from the visible solar disk by $S D O /$ AIA. There is a hint of detection by GOES of a smaller-than-C-class flare. However, the peak rate of $2.5 \times 10^{5}$ photons $\mathrm{s}^{-1}$ detected by STEREO-B in its $195 \AA$ channel indicates that the flare would have been classified as GOES X3.5 had it not been occulted (Nitta et al. 2013). LASCO detected a halo CME with a first C2 appearance at 08:00:05 UT with a linear speed of $1400 \mathrm{~km} \mathrm{~s}^{-1}$.

Upon exiting the South Atlantic Anomaly (SAA $)^{54}$ at 07:55:00 UT, both instruments on board Fermi detected emission associated with this flare. Fermi-LAT detected $>100 \mathrm{MeV}$ emission for approximately 20 minutes (with no evidence of temporally extended emission after 08:15:00 UT), and Fermi-GBM detected emission in the tens of keV range. RHESSI detected emission starting at $\sim 08: 18: 00$ UT (upon exiting spacecraft night) also in the tens of keV energy range for over 40 minutes. Konus-Wind had a full view of the flare and detected emission only in the softest energy band, 20-78 keV, starting at 07:43:00 UT. Radio data above $1.3 \mathrm{GHz}$ from RSTN for this flare did not indicate a detection. The GOES, RHESSI, Konus-Wind, Fermi-GBM, and FermiLAT LCs for this flare are shown in Figure 3.

SOL2014-09-01: On 2014 September 1 (Sep14), between 10:55:56 and 11:00:56 UT, a bright solar flare occurred in an AR located at N14E126. There was no GOES signal but STEREO-B had an unblocked view of the entire flare and detected a maximum rate of $1.7 \times 10^{7}$ photons $\mathrm{s}^{-1}$ in its $195 \AA$ channel, indicating an unocculted GOES X2.4 class flare (Nitta et al. 2013). LASCO detected a halo CME with first C2 appearance at 11:12:05 UT with a linear speed of $1900 \mathrm{~km} \mathrm{~s}^{-1}$. A type II radio burst with an estimated velocity of $2079 \mathrm{~km} \mathrm{~s}^{-1}$ was reported by NOAA Space Weather Alerts in association with this flare. SDO/AIA reported a coronal wave from this AR starting during 10:45:35-12:21:35 UT. This wave was seen to propagate along the limb and over onto the visible disk. ${ }^{55}$

\footnotetext{
${ }^{54}$ Both detectors on board Fermi are turned off while the spacecraft is in the SAA.

55 As reported by Nitta on http://www.lmsal.com/isolsearch.
} 


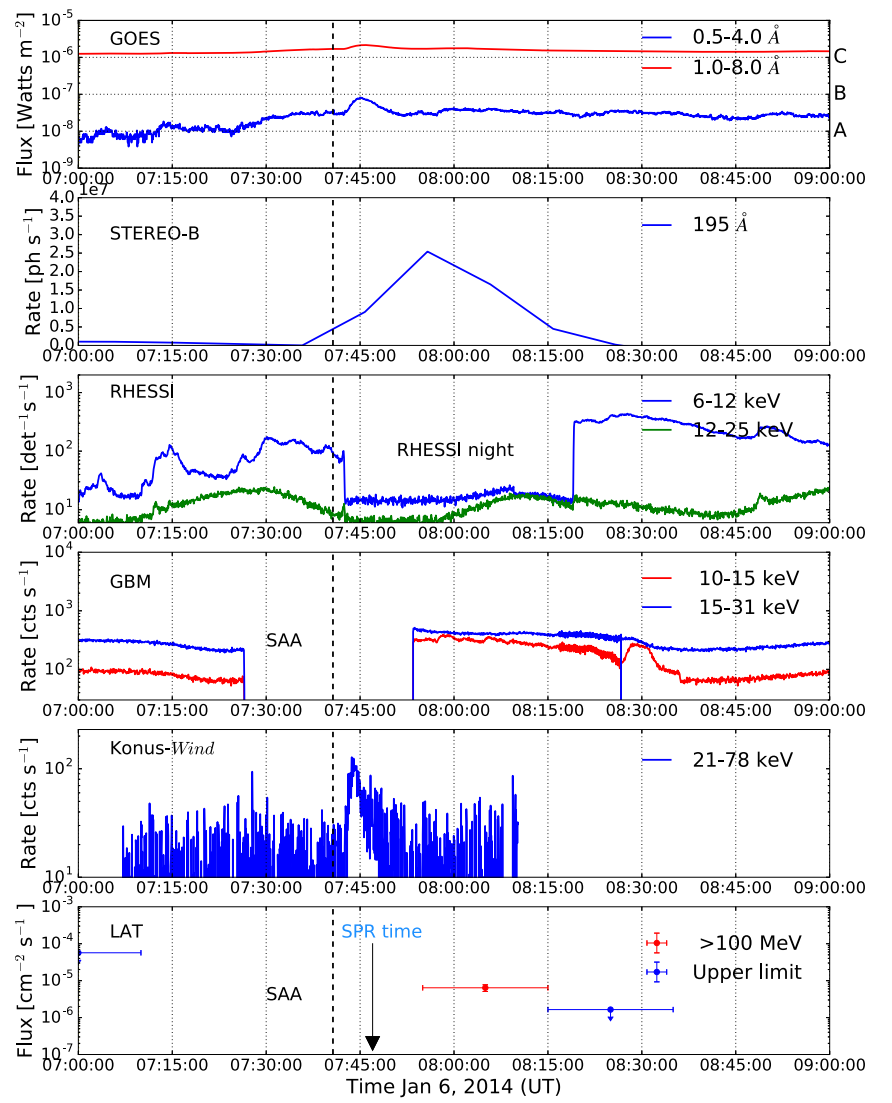

Figure 3. Composite light curve for Jan 14 with data from GOES, STEREO, RHESSI, Fermi-GBM, Konus-Wind, and Fermi-LAT. The vertical dashed line represents the estimated start time of the flare, 07:40:50 UT based on STEREO$\mathrm{B}$ emission. The time of Solar Particle Release (SPR) in the solar atmosphere is described in Section 4.

Fermi-LAT detected emission from this flare for $\sim 2 \mathrm{hr}$, peaking between 11:10:00-11:15:00 UT. ${ }^{56}$ The GBM detected emission up to a few $\mathrm{MeV}$ in temporal coincidence with the Fermi-LAT emission in both the BGO and NaI detectors. RHESSI was in the SAA from 10:55:00 to 11:11:00; upon exiting the SAA it detected emission up to $30 \mathrm{keV}$. KonusWind detected emission in all three energy bands, $20-78 \mathrm{keV}$, $78-310 \mathrm{keV}$, and $310-1180 \mathrm{keV}$, in temporal coincidence with Fermi-GBM. A significant radio flux at frequencies up to $16 \mathrm{GHz}$ was detected by the San Vito station of the RSTN simultaneously with the HXR emission peak detected by both Fermi-GBM and Konus-Wind. Figure 4 shows the LCs from GOES, STEREO, RHESSI, Fermi-GBM, Konus-Wind, and Fermi-LAT. The LCs of the microwave emission (MW) are compared with the HXR LC from Konus-Wind in Figure 5. The MW intensity distribution with frequency has a maximum at about $1 \mathrm{GHz}$ (see Section 3.4). Thus, we show the time profile of only this frequency for this flare. As evident from Figure 5, there is good agreement between these LCs.

\subsection{Localization of the Emission}

We present composite images of the Sun as seen by STEREO and SDO and, whenever available, the position of HXR emission based on RHESSI and the $>100 \mathrm{MeV}$ gamma-

\footnotetext{
56 Thanks to improvements provided by the new Fermi-LAT event selection, we gained 10 extra minutes of coverage with respect to Pass7_REP.
}
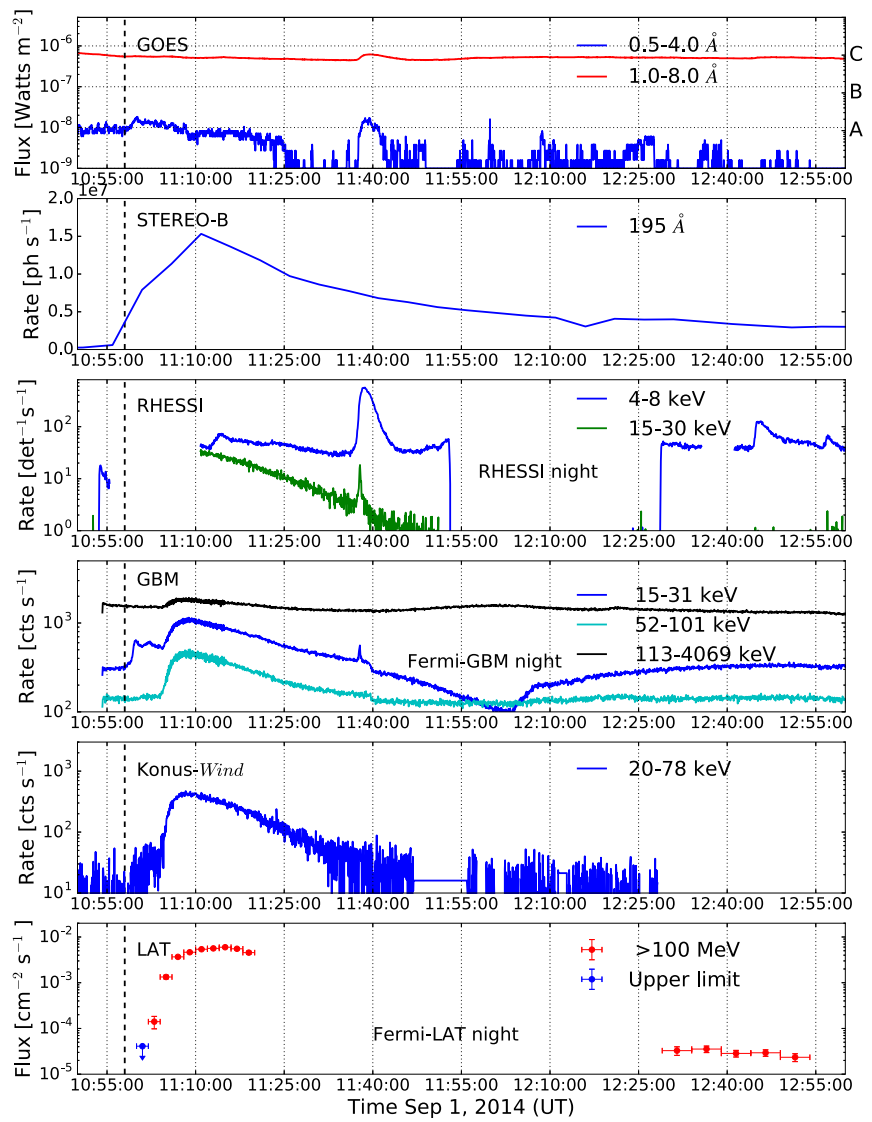

Figure 4. Light curves of Sep14 as seen by GOES, STEREO, RHESSI, FermiGBM (black line is for the BGO detector while cyan and blue are for $\mathrm{NaI}$ ), Konus-Wind, and Fermi-LAT. The vertical dashed line represents the estimated starting time of the flare, 10:58:00 UT based on STEREO-B emission. FermiLAT night was from $\sim 11: 35-12: 20$ UT. The increase in rate seen in RHESSI and Fermi-GBM at 11:38 UT is due to a small on-disk flare.

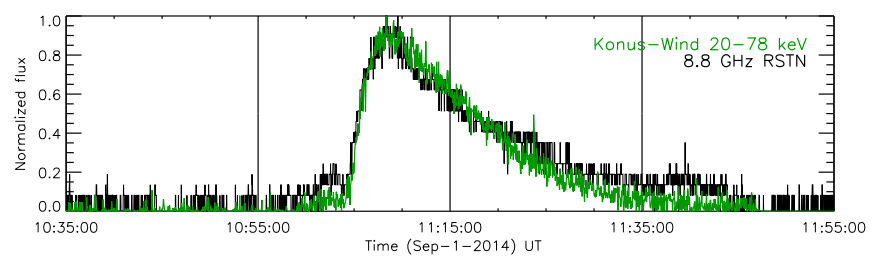

Figure 5. Comparison of the 20-78 keV HXR emission and MW light curves at $8.8 \mathrm{GHz}$ (optically thin gyro-synchrotron emission) for the Sep14 flare. Profiles are normalized to the peak value.

ray emission centroid based on Fermi-LAT data. We used the FITS World Coordinate System software package (Thompson $\&$ Wei 2010) to co-register the locations of the flares between STEREO and SDO images. We applied the CLEAN imaging algorithm (Hurford et al. 2002) to RHESSI data using detectors 3-9 to reconstruct the X-ray images. The centroid for the $>100 \mathrm{MeV}$ gamma-ray emission is determined using the gtfindsrc tool, which performs a likelihood analysis of the average position in the time-integrated data set.

Figure 6 displays the composite image for the Oct13 flare showing the STEREO-B $195 \AA$ image of the AR located about $10^{\circ}$ behind the limb, the SDO/AIA $193 \AA$ emission peaking above the limb, a contour of the RHESSI image, and results from reanalysis of the flare with Pass 8 data. The new emission centroid is located in heliocentric coordinates [-880", 290"], about $200^{\prime \prime}$ closer to the RHESSI centroid, and with a $68 \%$ error 


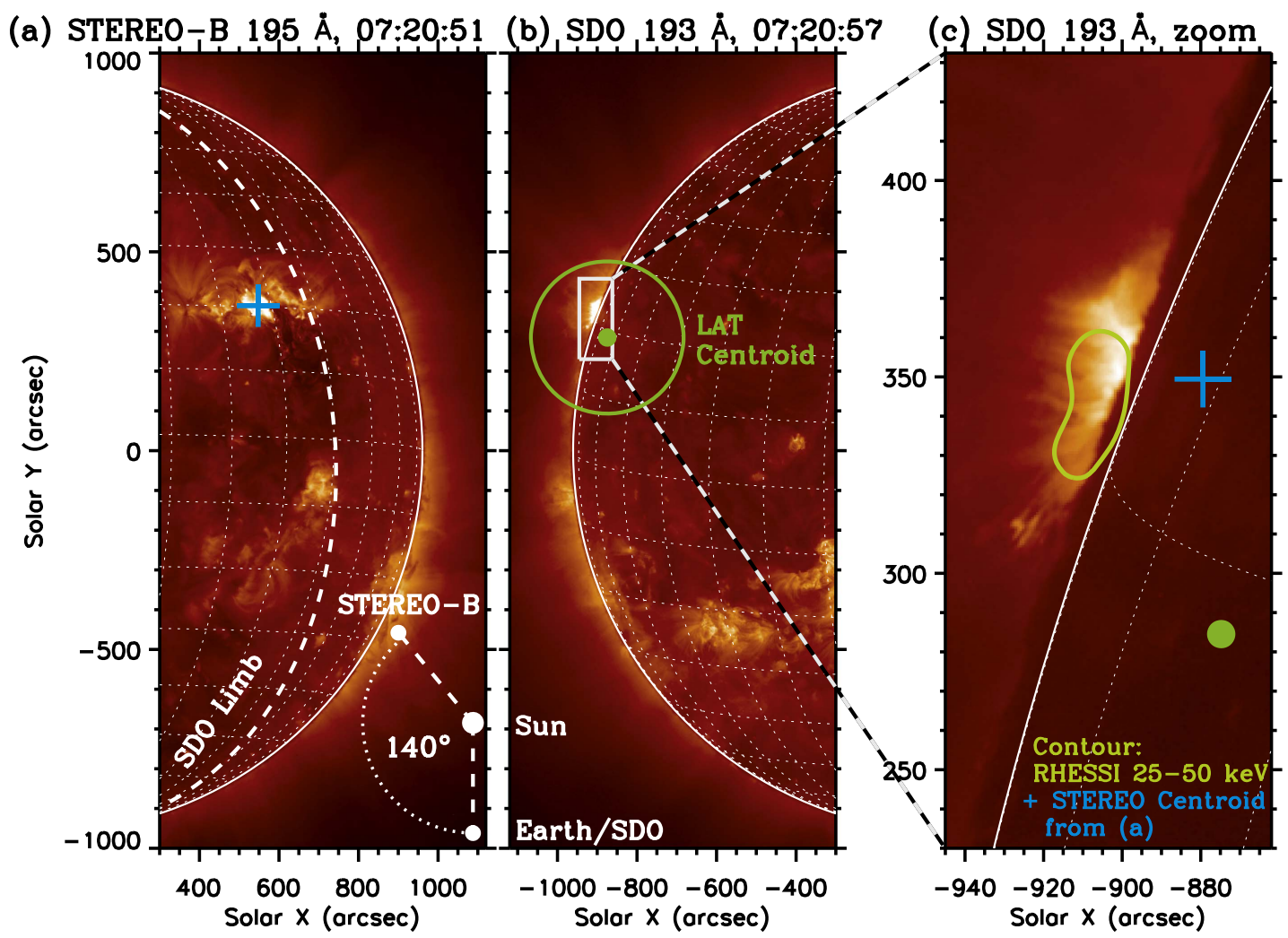

Figure 6. Localization of the Oct13 flare. Images near the flare peak are shown for STEREO-B $195 \AA$ (a), SDO $193 \AA$ (b), and an enlargement of the SDO image (c) marked by the white rectangle in (b). The green circle in (b) shows the $68 \%$ error circle for the Fermi-LAT emission centroid and the green dot in (c) represents the Fermi-LAT emission centroid position. The time range for the Fermi-LAT emission is from 07:10:00-07:35:00 UT. The green contour in (c) shows the 25-50 keV RHESSI source. The blue cross in (a) and (c) marks the centroid of the STEREO flare ribbon as seen from the STEREO and Earth/SDO perspectives, respectively. The white dashed line in (a) represents the solar limb as seen by SDO. The positions of STEREO and SDO/Earth relative to the Sun are shown in the lower-right corner of (a).

radius of $190^{\prime \prime}$, which is $\sim 20 \%$ smaller than the value we reported in Pesce-Rollins et al. (2015) using Pass7_REP data. In addition, in the Pass 8 data set, the total number of $>1 \mathrm{GeV}$ events measured from this flare increased from four to seven. The highest-energy photon detected from this flare was $3.4 \mathrm{GeV}$ and the arrival was 07:19:00 UT.

For the Jan14 flare, the Fermi-LAT photon statistics were not sufficient to provide an emission localization error circle smaller than 0.5 . However, we can still conclude that the emission detected by Fermi-LAT was consistent with the position of the Sun. In Figure 7 we show the STEREO-A and $S D O$ images of this event at two different times. The top panels of Figure 7 present SDO $171 \AA$ (left) and STEREO-A $195 \AA$ (right) image at 07:55:46 UT and show the filament eruption, while the bottom panels show the SDO $193 \AA$ (left) and STEREO-A $195 \AA$ (right) images at 08:25:46 UT with the RHESSI 6-12 and 25-50 keV contours of this flare.

The Fermi-LAT $>100 \mathrm{MeV}$ emission centroid of Sep14 is located at heliocentric coordinates $\left[-720^{\prime \prime}, 610^{\prime \prime}\right]$ with a $68 \%$ error radius of $100^{\prime \prime}$. RHESSI imaging shows a $6-12 \mathrm{keV}$ source located above the visible limb slightly offset from the Fermi-LAT centroid, both shown in Figure 8. If the RHESSI source is the loop top of the BTL flare, then the minimum height needed for this source to be visible from $\sim 40^{\circ}$ BTL would be $\sim 10^{10} \mathrm{~cm}$. HXR loop-top emission from a flare located $\sim 40^{\circ}$ BTL has been detected before by RHESSI (Krucker et al. 2007). Fermi-LAT measured 17 photons with energies $>1 \mathrm{GeV} ; 15$ of these (including a
3.5 GeV photon with an arrival time of 11:16:01 UT) arrived during the first 20 minutes of Fermi-LAT detection.

\section{Spectral Analysis}

\subsection{Gamma-Ray Spectra}

We performed an unbinned likelihood analysis of the FermiLAT data with the gtlike program distributed with the Fermi ScienceTools. ${ }^{57}$ We selected Pass 8 Source class events from a $10^{\circ}$ circular region centered on the Sun and within $100^{\circ}$ from the local zenith (to reduce contamination from the Earth limb).

We fit three models to the Fermi-LAT gamma-ray spectral data. The first two, a pure power law (PL) and a power law with an exponential cutoff (PLEXP), are phenomenological functions that may describe bremsstrahlung emission from relativistic electrons. The third model uses templates based on a detailed study of the gamma-rays produced from the decay of pions originating from accelerated protons with an isotropic pitch angle distribution in a thick-target model (updated from Murphy et al. 1987).

We rely on the likelihood ratio test and the associated test statistic TS (Mattox et al. 1996) to estimate the significance of the detection. The TS is defined as twice the increment of the logarithm of the likelihood obtained by fitting the data with the source and background model components simultaneously.

\footnotetext{
${ }^{57}$ We used the version 10-01-00 available from the Fermi Science Support Center: http://fermi.gsfc.nasa.gov/ssc/.
} 


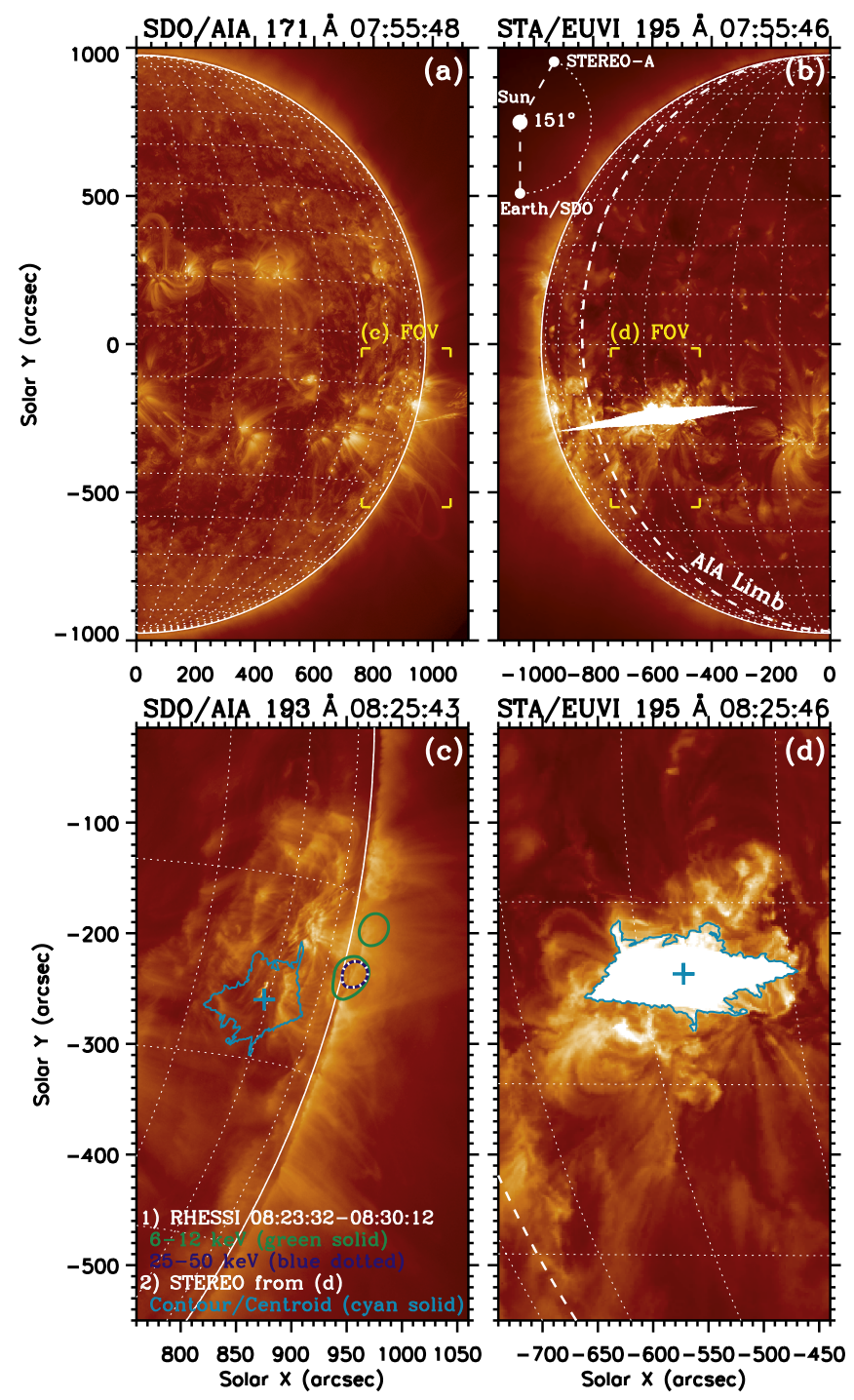

Figure 7. SDO 171 and $193 \AA$ (left) and STEREO-B $195 \AA$ (right) images near the Jan14 flare peak. The white dashed line in (b) and (d) represents the solar limb as seen by $S D O$. The cyan contour and cross in (d) mark the STEREO flare ribbon and its centroid, respectively. Their projected view as seen in the AIA perspective is shown in (c), in which the centroid is located $20^{\circ}$ behind the western limb. The green solid and blue dotted contours in (d) show the RHESSI 6-12 and 25-50 keV sources, respectively. The rectangular brackets in (a) and (b) mark the field of view for (c) and (d), respectively.

Because the null hypothesis (i.e., the model without an additional source) is the same for the PL and PLEXP models, the increment of the TS $\left(\triangle \mathrm{TS}=\mathrm{TS}_{\mathrm{PLEXP}}-\mathrm{TS}_{\mathrm{PL}}\right)$ is equivalent to the corresponding difference of the maximum likelihoods computed between the two models. Note that the significance in $\sigma$ can be roughly approximated as $\sqrt{\mathrm{TS}}$ for two degrees of freedom.

In Table 1 we list the $\mathrm{TS}_{\mathrm{PL}}, \Delta \mathrm{TS}$, photon index $\Gamma$ for the best-fit model (PL when $\triangle \mathrm{TS}<25$ or PLEXP when $\Delta \mathrm{TS} \geqslant 25$ ), and PLEXP cutoff energy. For several intervals, $\Delta \mathrm{TS}>25$, indicating that PLEXP provides a significantly better fit than PL. For these intervals we fit the pion-decay models to the data to determine the best proton spectral index following the same procedure described in Ajello et al. (2014). In particular, we performed a series of fits with the pion-decay template models calculated for a range of proton spectral indices. We then fit the resulting profile of the log-likelihood with a parabolic function of the proton index. The minimum gives the most likely index for the pion-decay model. Note that the TS values for the PLEXP and pion-decay fits cannot be directly compared (Wilks 1938) because they are not nested models. However, PLEXP approximates the shape of the piondecay spectrum; thus, we expect the pion-decay models to provide a similarly acceptable fit.

The main contributions to the systematic uncertainties are uncertainties in the effective area; in the energy range $100 \mathrm{MeV}$ to $\sim 100 \mathrm{GeV}$, these are of the order of $\pm 5 \%$. This uncertainty applies directly to the flux values and from our previous studies on LAT-detected solar flares (Ajello et al. 2014) we find that the systematic uncertainties on the cutoff energy and photon index are also of the order of $\pm 5 \%$. $^{58}$

\subsubsection{Comparison of BTL and Disk Flare Characteristics}

We compare the characteristics of the $>100 \mathrm{MeV}$ emission associated with the three BTL flares with three disk flares with similar GOES classifications and temporally extended emissions and are described in Ackermann et al. (2014) and Ajello et al. (2014). In addition to spectral parameters, we also compare the total $>100 \mathrm{MeV}$ emitted energies, and the total energy released by protons with energy $>500 \mathrm{MeV}$ needed to produce the detected gamma-ray emission, based on the templates of (updated from) Murphy et al. (1987). We present these numbers along with the date, estimated GOES class, CME speed, AR position, and Fermi-LAT detection duration in Table 2 . The proton indexes are very similar whereas the ondisk flares appear to have more energy; this is most likely because we observed the on-disk flares over longer timescales. Peak fluxes and the total energy released by protons with $E>500 \mathrm{MeV}$ for Sep14 and the impulsive phase of SOL201203-07 are comparable.

Figure 9 shows the proton index as a function of time for the Oct13 and Sep14 BTL flares. We fit a constant (red dashed line) and a first-degree polynomial (blue dashed line) to the data. In the case of the constant fit, we list the best-fit value in the upper left corner, whereas for the straight-line fit, we list the value of the slope. The temporal variation over tens of minutes is not sufficient to conclude whether a softening or hardening is present for these BTL flares. In Ajello et al. (2014) we found that for the on-disk flare SOL2012-03-07 the spectrum softened with a timescale of a few hours.

\subsection{X-Ray Spectra}

As described above we have HXR data from three instruments: RHESSI, Fermi-GBM, and Konus-Wind. KonusWind was in waiting mode during the Jan14 and Sep14 flares; therefore, these events were detected in only three broad energy channels, namely $\sim 20-78,78-310$, and 310-1180 keV, with $2.944 \mathrm{~s}$ time resolution. RHESSI provides only a limited coverage of these flares. For the Oct13 flare there is some overlap with Fermi-GBM during the rise of the impulsive phase. As shown in Pesce-Rollins et al. (2015) the HXR spectra of RHESSI and Fermi-GBM agree well. Fermi-GBM was in the SAA and RHESSI was in spacecraft night during the impulsive phase of the Jan14 flare and detected the flare during the decay phase in only two low-energy channels. Thus, we do

\footnotetext{
58 A more detailed description of the systematic uncertainties tied to the effective area of the LAT can be found here: http://fermi.gsfc.nasa.gov/ssc/ data/analysis/LAT_caveats.html.
} 


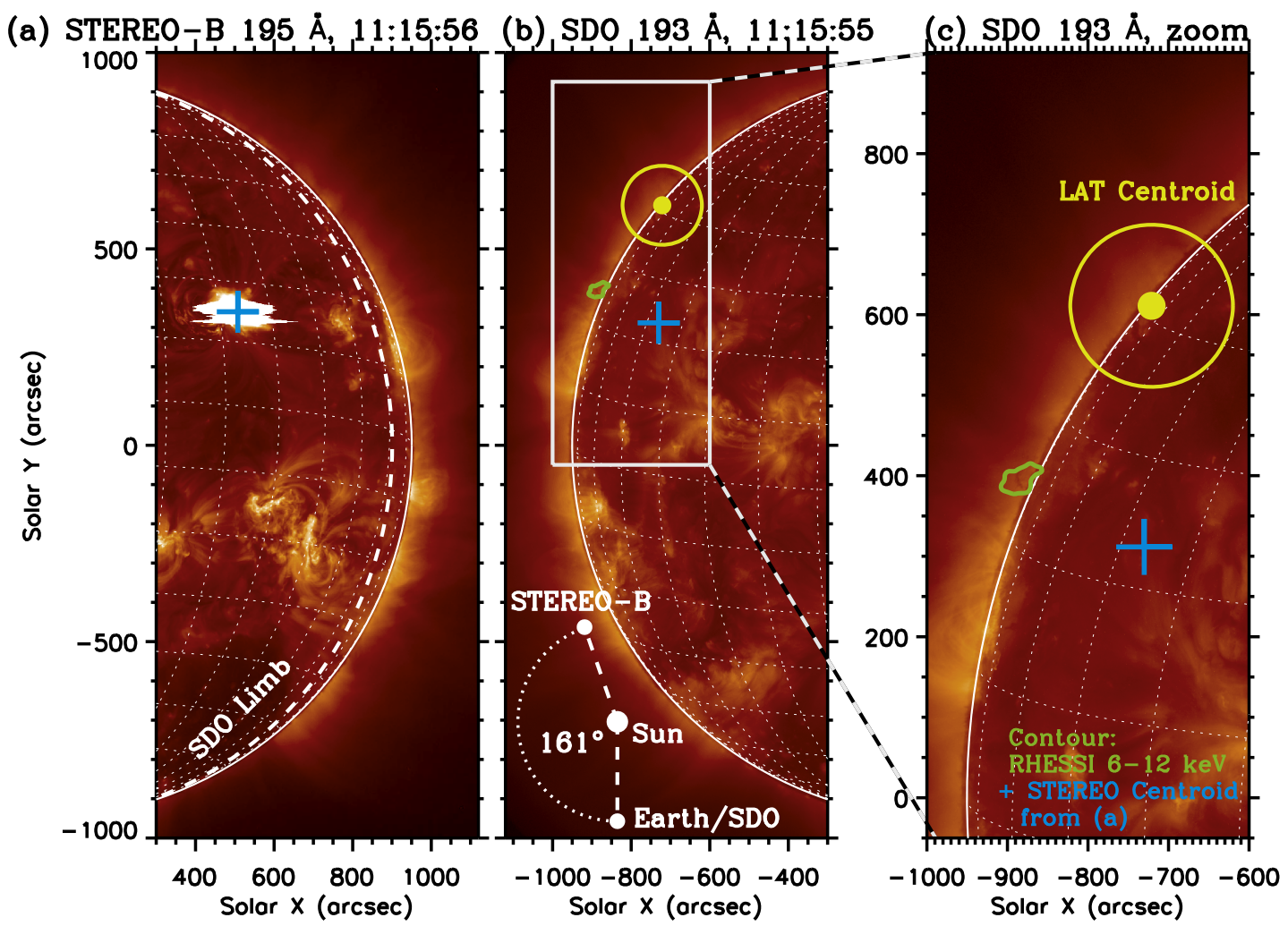

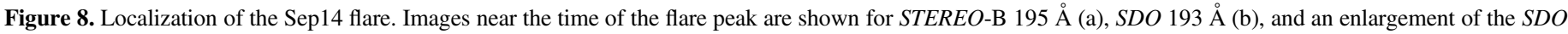

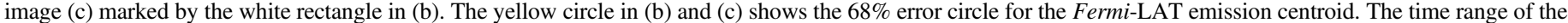

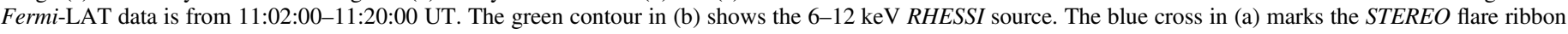

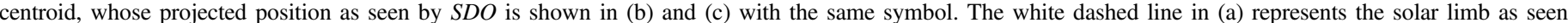
by $S D O$.

not have sufficient data for a spectral fit. For the Sep14 flare, RHESSI gives information only on the decay phase of the flares. In the following we show results from the analysis of the Fermi-GBM data for the Oct13 and Sep14 flares.

\subsection{Combined Spectroscopic Studies}

For both Oct13 and Sep14, we performed a combined Fermi-GBM/LAT fit using the XSPEC package (Arnaud 1996). The spectral fits were done by minimizing PGSTAT, a profile likelihood statistic that takes into account Poisson error on the total count spectrum and Gaussian error on the background. In order to obtain the background-subtracted spectra of the FermiGBM data for Sep14, we used both Fermi-GBM-NaI and Fermi-GBM-BGO spectra accumulated before the flare (from 10:54 UT to 10:57 UT) and after the flare (from 11:42-11:50 UT). For Oct13, because there was a minor on-disk flare whose onset time was 7:01 UT, we used the procedure described in Pesce-Rollins et al. (2015), which consists of using the background estimation tool developed in Fitzpatrick et al. (2012) with an additional 5\% systematic error. For the LAT, we follow the procedure used in Ajello et al. (2014) and PesceRollins et al. (2015) that consists of deriving the background spectrum directly from the model of the background used in the standard LAT likelihood analysis (first using gt $\mathrm{i}$ ke and then gtbkg), and obtaining the response of the LAT using gtrspgen(all of these tools are available in the Fermi ScienceTools). The combined spectral energy distribution (SED) derived from the Fermi-GBM and Fermi-LAT data extending from $30 \mathrm{keV}$ to $10 \mathrm{GeV}$ for these flares is shown in Figures 10 and 11, respectively, and the parameters for the spectral fit for both solar flares are listed in Table 3.

The Oct13 flare had a very weak signal in the BGO, and the best-fit model $\left(\mathrm{M}_{0}\right)$ consisted of a single PL and the pion-decay templates to describe the bremsstrahlung and $>60 \mathrm{MeV}$ emission detected by Fermi-GBM and Fermi-LAT, respectively. For the Sep14 flare, the best-fit model $\left(\mathrm{M}_{0}\right)$ consisted of a single PL with an exponential cutoff at high energies to describe the bremsstrahlung emission, and the pion-decay templates to describe the $>60 \mathrm{MeV}$ emission detected by Fermi-LAT, similar to what was done for the 2010 June 12 Fermi flare (Ackermann et al. 2012). We also tested the statistical significance of an extra PL with a high-energy cutoff (model $\mathrm{M}_{1}$ ). To this end, we performed Monte Carlo simulations (using XSPEC fakeit) by generating a spectrum described by the best-fit model $\left(\mathrm{M}_{0}\right)$. We then re-optimized the parameters of $\mathrm{M}_{0}$ by fitting it to the simulated data. We finally compared the improvement of PGSTAT when fitting the simulated data with the model $\mathrm{M}_{1}$. The improvement of PGSTAT for the Oct13 and Sep14 flares is $\Delta_{\mathrm{PGSTAT}} \approx 408$ and $\approx 109$, respectively. Despite these high values of $\Delta_{\text {PGSTAT }}$, dedicated Monte Carlo simulations indicate that they correspond to significances of $2.5 \sigma$ and $2.0 \sigma$, respectively.

We then checked whether the addition of the $2.223 \mathrm{MeV}$ line (on top of the model $\mathrm{M}_{0}$ ) significantly improved the fit. For the Oct13 flare, the improvement was negligible $\left(\Delta_{\mathrm{PGSTAT}} \approx 0\right)$, while for the Sep14 the improvement was $\Delta_{\mathrm{PGSTAT}} \approx 25$, corresponding to a significance of $2.0 \sigma$, estimated from Monte Carlo simulations. We tested whether adding nuclear deexcitation narrow lines and continua provided an improvement 
Table 1

Fermi-LAT Spectral Analysis of the Solar Flares Considered in this Work

\begin{tabular}{|c|c|c|c|c|c|c|}
\hline $\begin{array}{l}\text { Time Interval } \\
\text { (UT) }\end{array}$ & $\mathrm{TS}_{\mathrm{PL}}$ & $\Delta \mathrm{TS}^{\mathrm{a}}$ & Photon Index ${ }^{\mathrm{b}}$ & $\begin{array}{c}\text { Cutoff Energy }{ }^{c} \\
(\mathrm{MeV})\end{array}$ & $\begin{array}{c}\text { Flux }^{\mathrm{d}} \\
\left(\times 10^{-5} \mathrm{ph} \mathrm{cm}^{-2} \mathrm{~s}^{-1}\right)\end{array}$ & Proton Index \\
\hline \multicolumn{7}{|c|}{ SOL2013-10-11 } \\
\hline $07: 10: 00-07: 15: 00$ & 20 & 3 & $-2.0 \pm 0.4$ & $\ldots$ & $0.9 \pm 0.1$ & $\ldots$ \\
\hline $07: 15: 00-07: 18: 00$ & 487 & 43 & $-0.4 \pm 0.4$ & $130 \pm 30$ & $17 \pm 1$ & $4.5 \pm 0.4$ \\
\hline 07:18:00-07:20:00 & 846 & 77 & $-0.1 \pm 0.4$ & $112 \pm 21$ & $49 \pm 2$ & $4.3 \pm 0.2$ \\
\hline $07: 20: 00-07: 22: 00$ & 776 & 76 & $-0.3 \pm 0.3$ & $128 \pm 23$ & $46 \pm 2$ & $4.4 \pm 0.3$ \\
\hline $07: 22: 00-07: 24: 00$ & 677 & 58 & $-0.2 \pm 0.4$ & $153 \pm 31$ & $29 \pm 2$ & $3.7 \pm 0.2$ \\
\hline $07: 24: 00-07: 26: 00$ & 345 & 25 & $-0.9 \pm 0.4$ & $208 \pm 61$ & $21 \pm 2$ & $3.9 \pm 0.3$ \\
\hline $07: 26: 00-07: 28: 00$ & 219 & 34 & $0.6 \pm 0.7$ & $86 \pm 22$ & $16 \pm 1$ & $4.5 \pm 0.4$ \\
\hline 07:28:00-07:40:00 & 283 & 29 & $0.1 \pm 0.7$ & $86 \pm 26$ & $7 \pm 1$ & $5.3 \pm 0.4$ \\
\hline \multicolumn{7}{|c|}{ SOL2014-01-06 } \\
\hline 07:55-08:15 & 67 & 20 & $-2.4 \pm 0.2$ & $\cdots$ & $0.6 \pm 0.1$ & $\cdots$ \\
\hline \multicolumn{7}{|c|}{ SOL2014-09-01 } \\
\hline 11:02:00-11:04:00 & 42 & 9 & $-2.5 \pm 0.3$ & $\cdots$ & $11 \pm 3$ & $\cdots$ \\
\hline 11:04:00-11:06:00 & 321 & 41 & $-0.2 \pm 0.5$ & $117 \pm 27$ & $117 \pm 10$ & $4.7 \pm 0.4$ \\
\hline 11:06:00-11:08:00 & 1070 & 120 & $-0.5 \pm 0.3$ & $116 \pm 15$ & $360 \pm 17$ & $5.2 \pm 0.2$ \\
\hline 11:08:00-11:10:00 & 1549 & 126 & $-0.9 \pm 0.2$ & $158 \pm 20$ & $477 \pm 19$ & $4.9 \pm 0.2$ \\
\hline $11: 10: 00-11: 12: 00$ & 2549 & 115 & $-1.2 \pm 0.2$ & $194 \pm 28$ & $565 \pm 21$ & $4.8 \pm 0.2$ \\
\hline $11: 12: 00-11: 14: 00$ & 6394 & 157 & $-0.8 \pm 0.1$ & $167 \pm 10$ & $522 \pm 20$ & $4.4 \pm 0.1$ \\
\hline 11:14:00-11:16:00 & 2430 & 159 & $-0.6 \pm 0.2$ & $148 \pm 17$ & $540 \pm 22$ & $4.5 \pm 0.2$ \\
\hline $11: 16: 00-11: 18: 00$ & 1069 & 32 & $-1.9 \pm 0.2$ & $488 \pm 90$ & $465 \pm 23$ & $4.2 \pm 0.2$ \\
\hline $11: 18: 00-11: 20: 00$ & 1047 & 49 & $-1.0 \pm 0.3$ & $182 \pm 37$ & $396 \pm 27$ & $4.5 \pm 0.2$ \\
\hline $12: 29: 00-12: 34: 00$ & 56 & 12 & $-2.2 \pm 0.2$ & $\cdots$ & $3 \pm 1$ & $\cdots$ \\
\hline $12: 34: 00-12: 39: 00$ & 133 & 3 & $-2.3 \pm 0.2$ & $\cdots$ & $3 \pm 1$ & $\ldots$ \\
\hline $12: 39: 00-12: 44: 00$ & 130 & 5 & $-2.2 \pm 0.2$ & $\ldots$ & $2 \pm 1$ & $\ldots$ \\
\hline $12: 44: 00-12: 49: 00$ & 97 & 11 & $-2.3 \pm 0.2$ & $\cdots$ & $2 \pm 1$ & $\ldots$ \\
\hline $12: 49: 00-12: 54: 00$ & 84 & 3 & $-2.4 \pm 0.2$ & $\ldots$ & $2 \pm 1$ & $\ldots$ \\
\hline
\end{tabular}

Notes.

${ }^{\mathrm{a}} \triangle \mathrm{TS}=\mathrm{TS}_{\mathrm{PLEXP}}-\mathrm{TS}_{\mathrm{PL}}$.

${ }^{\mathrm{b}}$ Photon index from the best-fit model. The PL is defined as $\frac{d N(E)}{d E}=N_{0} E^{\Gamma}$ and the PLEXP as $\frac{d N(E)}{d E}=N_{0} E^{\Gamma} \exp \left(-\frac{E}{E_{c}}\right)$, where $E_{c}$ is the cutoff energy.

${ }^{\mathrm{c}}$ From the fit with the PLEXP model.

d Integrated flux between $100 \mathrm{MeV}$ and $10 \mathrm{GeV}$ calculated for the best-fit model.

to the fit for both flares and found that it was not significant. The nuclear line and continua templates used here are based on a detailed study of the nuclear gamma-ray production from accelerated ion interactions with elements found in the solar atmosphere (Murphy et al. 2009).

\subsection{Radio Spectra}

The radio spectra obtained at the peaks of the Oct13 and Sep14 events are shown in Figure 12. The spectrum was obtained by using the RSTN one-second resolution fluxes. The spectra were background subtracted and the fluxes were integrated within the selected time intervals for each frequency. For each spectrum we detected the frequency where the maximum flux was observed. The spectra of the Oct13 and Sep14 flares are similar to those expected from the gyrosynchrotron (GS) mechanism, with peaks that separate the optically thin and thick parts (for GS spectrum properties see, for example, Dulk 1985). The values for the microwave photon spectral index, $\alpha$, for both flares are shown in Figure 12. Unfortunately, the RSTN data for the time period of the Jan 14 flare are not sufficient to obtain radio spectra.

\section{Solar Energetic Particles}

Interpretation of the origin of SEPs is more complicated than studying photons, since they move with different velocities along curved magnetic field lines and are detected only when the field lines are connected to the instrument. Moreover, they may be scattered by turbulence with energy-dependent mean free path. The GOES proton fluxes did not show a significant increase for the Oct13 event (which occurred behind the eastern limb). However, STEREO-B, positioned behind the limb and with better magnetic connectivity, detected an increase of SEP proton intensity starting roughly $2 \mathrm{hr}$ after the detection of an electromagnetic signal from this flare. The Sep14 flare, however, which was located roughly $40^{\circ}$ behind the eastern limb, was associated with an increase in the proton fluxes detected both by STEREO-B (which had a front view of the flare) and GOES (starting roughly $9 \mathrm{hr}$ after the flare).

The Jan 14 flare, originating in an AR $20^{\circ}$ behind the western limb, produced no significant increase in the proton flux at STEREO, had better magnetic connectivity to the Earth, and was associated with a very strong SEP event with neutrons detected on the ground by the South Pole neutron monitors.

The arrival time $T_{2}$ (Debrunner et al. 1988) at the Earth of a particle with velocity $v=\beta c$ released from the Sun at time $T_{1}$, 
Table 2

Comparison Between Behind-the-limb and On-disk Flare Quantities

\begin{tabular}{|c|c|c|c|c|c|c|c|c|}
\hline $\begin{array}{l}\text { Date } \\
\text { (UTC) }\end{array}$ & $\begin{array}{l}\text { GOES }^{\mathrm{a}} \\
\text { Class }\end{array}$ & $\begin{array}{l}\text { CME Speed } \\
\left(\mathrm{km} \mathrm{s}^{-1}\right)\end{array}$ & $\begin{array}{l}\text { AR } \\
\text { Position }\end{array}$ & $\begin{array}{c}\text { Duration } \\
\text { (minutes) }\end{array}$ & $\begin{array}{c}\text { Peak Flux } \\
\left(10^{-5} \mathrm{ph} \mathrm{cm}^{-2} \mathrm{~s}^{-1}\right)\end{array}$ & $\begin{array}{c}E_{\gamma}>\underset{(e r g)}{100 \mathrm{MeV}^{\mathrm{d}}} \\
\end{array}$ & $\begin{array}{l}\text { Proton }^{\mathrm{e}} \\
\text { Index }\end{array}$ & $\begin{array}{c}E_{p}>500 \mathrm{MeV}^{\mathrm{f}} \\
(\mathrm{erg})\end{array}$ \\
\hline 2013-10-11 & M4.9 & 1200 & N21E103 & 30 & $49 \pm 2$ & $1.5 \times 10^{23}$ & $4.3 \pm 0.1$ & $9.8 \times 10^{24}$ \\
\hline 2014-01-06 & $\mathrm{X} 3.5$ & 1400 & S8W110 & 20 & $0.8 \pm 0.1$ & $4.2 \times 10^{21}$ & $5.3 \pm 0.4^{\mathrm{g}}$ & $3.5 \times 10^{23}$ \\
\hline \multicolumn{9}{|c|}{ On-disk flares } \\
\hline 2011-03-07 & M3.7 & 2125 & N30W48 & 798 & $3 \pm 1$ & $5.1 \times 10^{23}$ & $4.7 \pm 0.2$ & $3.6 \times 10^{25}$ \\
\hline $2012-03-07 \mathrm{E}^{\mathrm{i}}$ & X5.4 & 2684 & N16E30 & 1068 & $97 \pm 2$ & $1.4 \times 10^{25}$ & $4.3 \pm 0.1$ & $9.0 \times 10^{26}$ \\
\hline
\end{tabular}

Notes.

${ }^{\text {a }}$ GOES class for BTL flares is estimated based on the STEREO $195 \AA$ flux.

${ }^{\mathrm{b}}$ Speed is the linear speed reported by the LASCO CME catalog.

${ }^{c}$ For photon energies $>100 \mathrm{MeV}$.

${ }^{\mathrm{d}}$ Total energy released in $>100 \mathrm{MeV}$ gamma-rays integrated over the time interval when $\Delta \mathrm{TS}>25$.

${ }^{\mathrm{e}}$ Proton index in the same time interval as (d).

f Total energy released by protons with $E>500 \mathrm{MeV}$ estimated over the same interval as in (d). Values may be underestimated for flares with centroids at heliocentric angles $>75^{\circ}$.

${ }^{\mathrm{g}}$ The $\Delta \mathrm{TS}$ value for this flare is 20 , so the improvement over a power law is marginal.

${ }^{\mathrm{h}}$ Impulsive phase of the flare, Fermi-LAT detection from 00:38:52-01:23:52 UT. Note that the peak of the GOES X-ray flare occurred $\sim 6$ minutes prior to Fermi orbital sunrise.

${ }^{\mathrm{i}}$ Extended phase of the flare, starting from 02:27 UT.
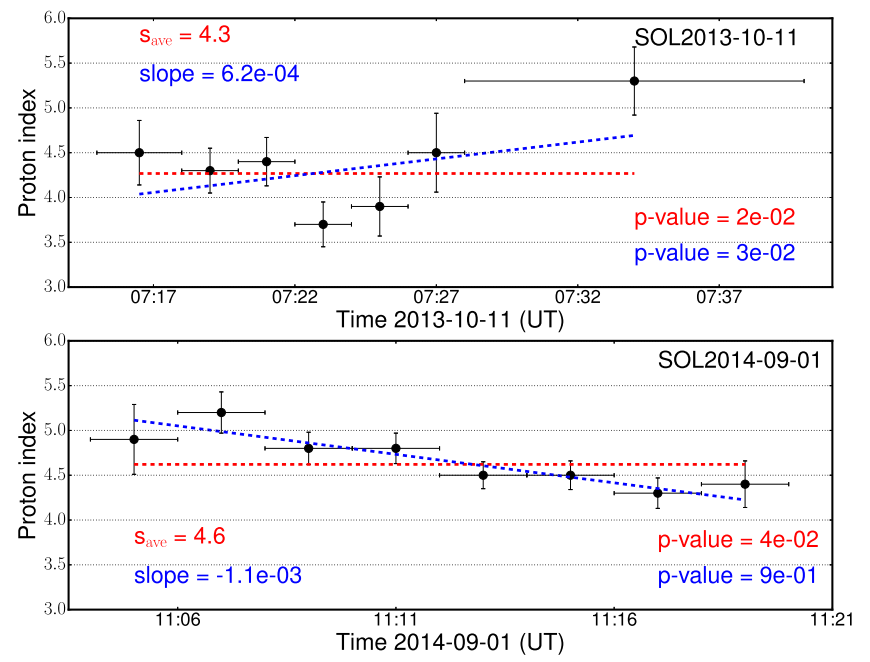

Figure 9. Proton index inferred from the fitting of the gamma-ray emission with the pion-decay templates as a function of time for Oct13 (top panel) and Sep14 (bottom panel). The red dashed lines represent a fit with a constant and the blue dashed lines a linear fit. Fit parameter values and $p$-values are indicated in each panel.

is

$$
T_{2}\left(\frac{1}{\beta}\right)=T_{1}+\frac{\zeta}{c} \times \frac{1}{\beta},
$$

where $\zeta$ is the distance traveled by the SEP from the acceleration region to the Earth. Assuming scatter-free propagation for the first-arriving particles, $\zeta$ will be independent of particle energy or velocity and will be equal to the path length along a Parker spiral magnetic field line from the Sun to the Earth (usually assumed to be $1.2 \mathrm{au}$ ). If all the particles are accelerated at the same place and time, then the intercept of the line fitting $T_{2}$ to $1 / \beta$ would give $T_{1}$, the time at which SEPs are

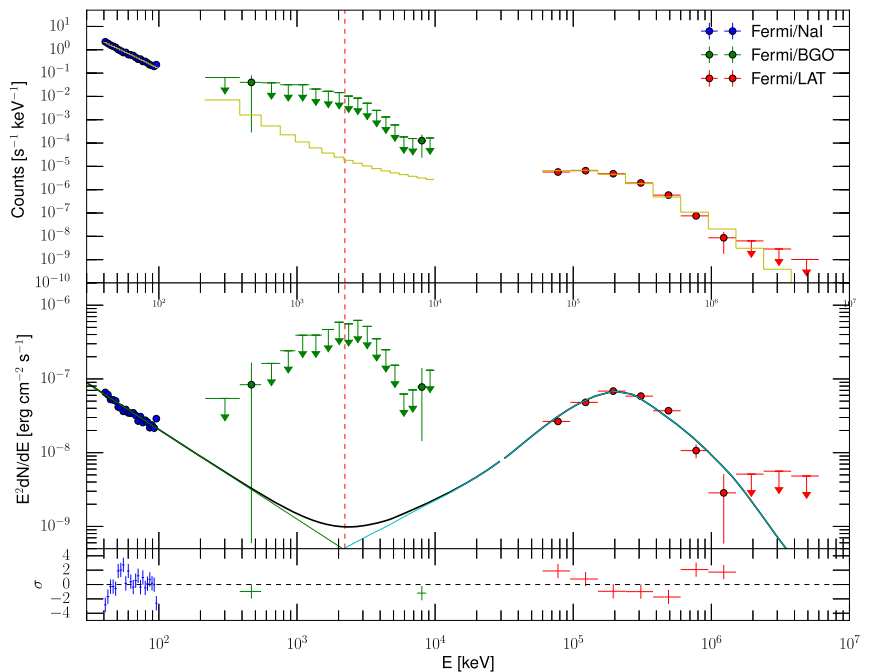

Figure 10. Combined Fermi-GBM/LAT count spectra (top panel) and spectral energy distribution (middle panel) for the Oct13 flare integrated between 7:10 and 7:35 UT; the lower panel shows the residuals of the fit. The models that best fit the data are a power law that dominates the low-energy spectrum and a pion-decay model, which describes the Fermi-LAT spectrum. The neutron capture line (at $2.223 \mathrm{MeV}$, highlighted by the red vertical dashed line) is not statistically significant, and neither is an additional low-energy power law with exponential cutoff $(\sim 2.5 \sigma)$. The parameters of the fits are listed in Table 3 .

released and is known as the Solar Particle Release (SPR) time. The slope of this line, $\zeta / c$, will be of the order of $600 \mathrm{~s}$.

Since we do not have high energy resolution SEP data we obtain the SEP onset times using two methods depending on the energy range of the particles. For the GOES SEPs with energies $<200 \mathrm{MeV}$ we apply a median filter to the intensity profile and define the onset time as when the profile reached $5 \%$ of the maximum. For the higher-energy SEPs we apply a Fast Fourier Transform (FFT) filter to the intensity profile and evaluate the time when the second derivative is maximum as 


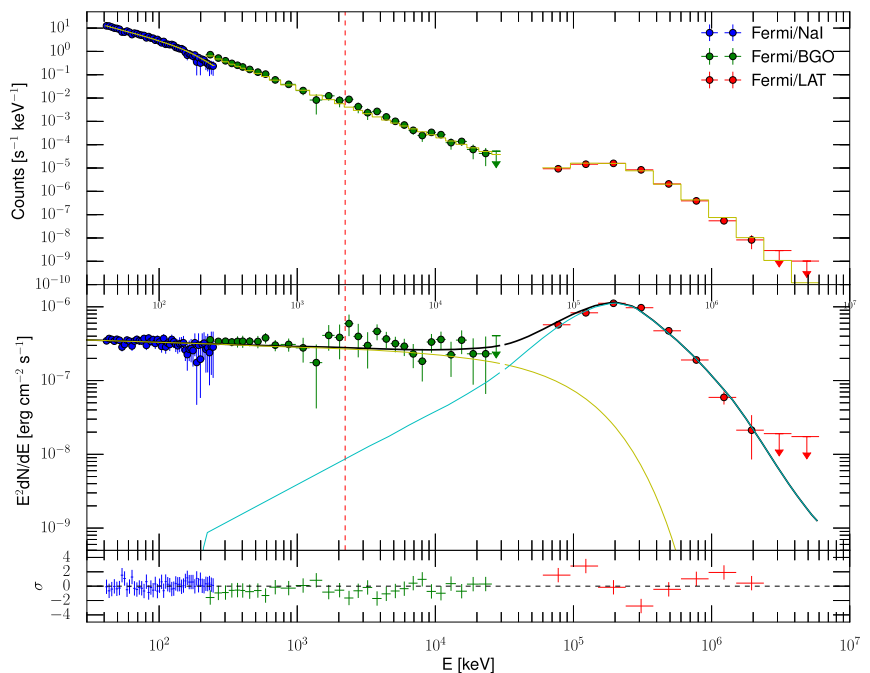

Figure 11. Combined Fermi-GBM/LAT count spectra (top panel) and SED (middle panel) for the Sep14 flare integrated between 11:02 and 11:20 UT; the lower panel shows the residuals of the fit. The best-fit models are a power law with exponential decay at high energy to describe the emission from $30 \mathrm{keV}$ to $\sim 10 \mathrm{MeV}$ and a pion-decay model to describe the Fermi-LAT spectrum. The neutron capture line (at $2.223 \mathrm{MeV}$, highlighted by the red vertical dashed line) is not statistically significant $(\sim 2 \sigma)$ and neither is an additional power law at low energy $\sim 2 \sigma$. The fit parameters are listed in Table 3.

Table 3

Best-fitting Spectral Parameters of the Fermi-LAT and Fermi-GBM Data

\begin{tabular}{lcc}
\hline \hline Parameter & & Value \\
\hline & SOL2013-10-11 & \\
\hline & Best-fit model: PL1+PION & \\
Power-law (1) index & & $3.2 \pm 0.1$ \\
Proton Index & PL1+PL2×EXP+PION & \\
& & $3.4 \pm 0.1$ \\
Power-law (1) index & & $-0.9 \pm 0.3$ \\
Power-law (2) index & & $0.8 \pm 0.1$ \\
Cutoff energy (MeV) & & $4.1 \pm 0.1$ \\
Proton Index &
\end{tabular}

SOL2014-09-01

Best-fit model: PL1 $\times$ EXP + PION

Power-law (1) index

Cutoff energy (MeV)

$2.06 \pm 0.01$

Proton Index

$90 \pm 7$

$4.4 \pm 0.1$

$(\mathrm{PL} 1+\mathrm{PL} 2) \times \mathrm{EXP}+\mathrm{PION}$

Power-law (1) index

Power-law (2) index

Cutoff energy $(\mathrm{MeV})$

$2.18 \pm 0.01$

$1.4 \pm 0.3$

$10 \pm 0.1$

Proton Index

$4.4 \pm 0.1$

Note. Model parameters for both the Oct13 and Sep14 flares. For the Oct13 flare we integrated between 7:10 and 7:35 UT, and for the Sep14 flare we integrated from 11:02 and 11:20 UT. The parameters of the best-fit model are compared with those of a more complex model with double power laws at low energy (whose improvement is not statistically significant).

the onset time. To estimate of the uncertainty on the onset time we performed a scan over a series of values for the median and FFT filter windows and take the difference in onset times as the error.

The onset times as a function of $\frac{1}{\beta}$ for the GOES SEP protons associated with Jan14 are shown in Figure 13. Fitting the data

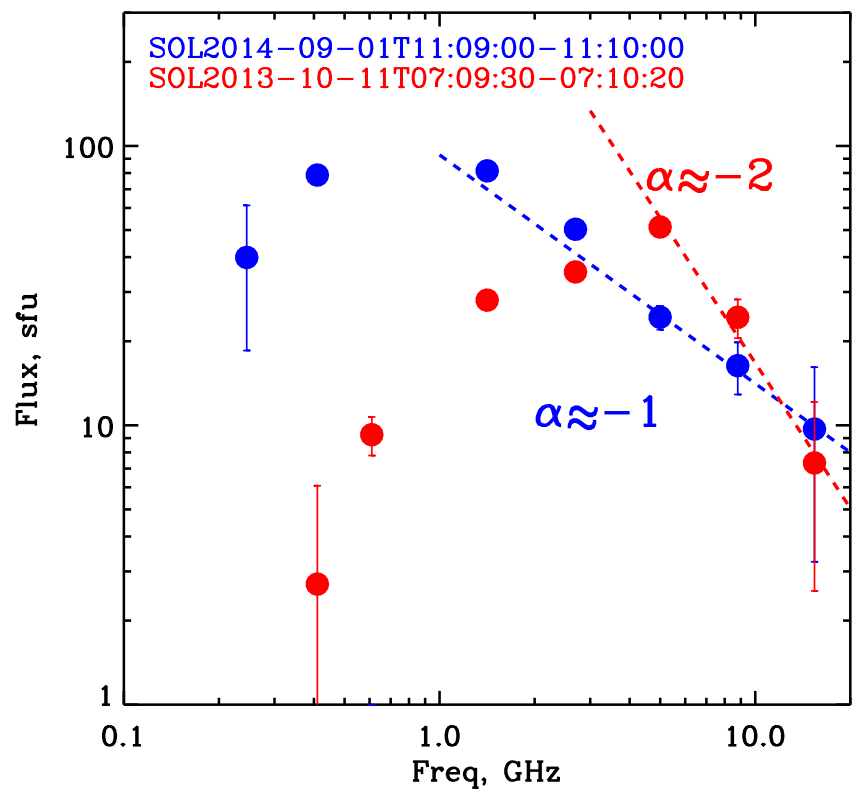

Figure 12. Radio spectra during the peaks of the HXR and radio time profiles (07:09:30-07:10:20 UT) for the Oct13 flare in red and for the Sep14 flare in blue (11:09:00-11:10:00 UT). The microwave photon spectral index $\alpha$, found by fitting the optically thin part of the spectra for each flare, is also shown.

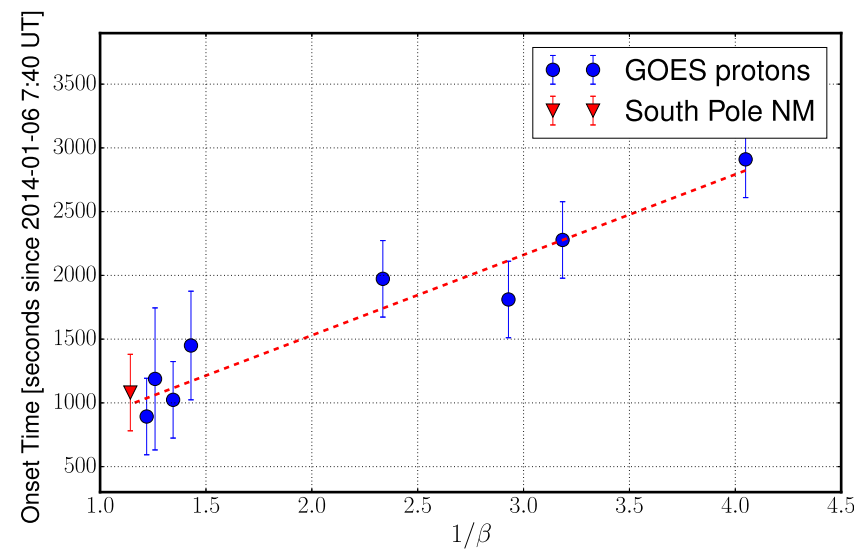

Figure 13. Onset times as a function of $1 / \beta$ for the GOES SEP protons associated with Jan14. The $Y$-axis gives the time in seconds since 2014-01-06 07:40 UT (assumed start of the flare as detected by STEREO-B) assuming scatter-free propagation for the first-arriving particles. The red dashed line represents the fit using Equation (1), giving the path length $\zeta=1.3 \pm 0.2$ au and $T_{1}=07: 55 \pm 00: 05 \mathrm{UT}$.

to Equation (1), we estimate the SPR time to be $T_{1}=07: 55 \pm 0: 05$ UT and the path length $\zeta \sim 1.3 \pm 0.2 \mathrm{au}$.

The value for $T_{1}$ is consistent with the estimated SPR time, 07:47 UT, reported by Thakur et al. (2014) but is somewhat later than the start of the flare activity at the Sun. Since Fermi was in the SAA and RHESSI was in orbital night, the most accurate time of the initiation of the flare was obtained from Konus-Wind and STEREO data. Konus-Wind detected emission in the 21-78 keV energy range starting at 07:43 UT, implying an emission time at the Sun of 07:35 UT, roughly 12 minutes prior to the SPR time. Assuming that the CME was released at this latter time, the acceleration of SEPs started when the CME was at a height of $1.5 R_{\odot}\left(v_{\mathrm{CME}} / 1400 \mathrm{~km} \mathrm{~s}^{-1}\right)$.

Unfortunately, the high-energy SEP data were not sufficient to estimate the SPR time for the other two BTL flares that occurred on Oct13 and Sep14. 


\section{Summary and Discussion}

We have presented the analysis of Fermi-LAT data from three BTL solar flares, whose location is determined by STEREO observations. We also presented analyses of data from RHESSI, Fermi-GBM, and Konus-Wind in the HXR range; $S D O$ in EUV; RSTN in radio wavelengths; some SEP observations; GOES; and the South Pole Neutron Monitors. We found several results.

1. The HXR LCs measured by the three instruments (in overlapping energy ranges) are in good agreement with each other and with the high-frequency radio LCs. The Fermi-LAT emission, in general, commences several minutes after the HXRs, peaks at a later time, and lasts longer.

2. We are able to obtain RHESSI images in soft HXRs for flares just over the limb, indicating a loop-top emission from loops with heights above the photosphere ranging from $0.2 \times 10^{10}$ to $2 \times 10^{10} \mathrm{~cm}$. SDO detected UV emission for Oct13, and possibly Jan14, but not for the Sep14 flare. The Jan14 flare was too weak to permit localization of the gamma-ray source, but localizations of the Fermi-LAT emission with Pass 8 data for the other two flares situates the centroid locations near the solar limb and about 50" (Oct13) and 300" (Sep14) from the RHESSI centroid.

3. The LCs (available only for the Oct13 and Sep14 flares) show good agreement between the HXR spectra measured by Fermi-GBM, RHESSI (whenever contemporaneous data are available), and Konus-Wind below a few $100 \mathrm{keV}$. The Fermi-LAT spectra can be fitted with a relatively hard PLEXP phenomenological model (possibly due to relativistic electron bremsstrahlung) or to a pion-decay model with proton indexes around 4 . We note that production of photons of about $\sim 3 \mathrm{GeV}$ detected here requires $>15 \mathrm{GeV}$ protons or $>6 \mathrm{GeV}$ electrons. These energies are greater than any other reported for solar particles.

4. We compare several spectral properties of $>100 \mathrm{MeV}$ radiation from the BTL flares with those of three on-disk flares previously analyzed. As evident from Table 2 and Figure 9, in general, these flares have similar characteristics. Similar GOES class flares have similar peak photon fluxes, surprisingly, regardless of limb occultation. The higher total gamma-ray energy of on-disk flares most likely results from their having been observed over longer timescales. Thus, the underlying acceleration and emission processes are most likely similar, but the transport paths of the radiating particles (presumably protons) from the acceleration to the emission site must be different. That is, for BTL flares, protons must travel greater distances to land on the front side of the Sun to produce the detected gamma-rays.

5. We find that the proton index does not vary significantly during the 30 minute observation time windows for the BTL flares. This is similar to that found for the on-disk flares for the same time frames, but some on-disk flares are detected over longer time intervals (up to $20 \mathrm{hr}$ ) during which the proton index increases gradually.

We now present our interpretation of these results with the goal of constraining the emission and acceleration mechanisms and sites.

\subsection{SEP Onset Times}

Since the spectral information available for the Jan14 flare is limited, we cannot constrain the acceleration, transport, or radiation processes. However, this flare is remarkable because the AR, being located beyond the western limb, provided good magnetic connection with the Earth and as a result it was associated with a very strong SEP event and increased the neutron flux in the South Pole neutron monitor. As presented in Figure 13, the acceleration time at the Sun for the SEP protons is 07:55 \pm 0:05 UT, which is in agreement with the SPR time of 07:47 \pm 0:08 UT reported by Thakur et al. (2014). Unfortunately, Fermi-LAT was in the SAA at the SPR time and exited at 07:55 UT. Therefore, we can only provide an upper limit on the time of acceleration of gamma-ray-producing particles (presumably protons) at the Sun. However, KonusWind detected emission in the $20-78 \mathrm{keV}$ band starting at 07:43 UT, implying that the electrons were accelerated at the Sun at 07:35 UT, roughly 12 minutes prior to the SPR.

Due to the location of the AR, the other two flares were not magnetically well connected to the Earth. For the Sep14 flare, the GOES proton fluxes begin to increase roughly $9 \mathrm{hr}$ after the flare start time. STEREO-B observed the flare in its entirety but provides proton fluxes only in three energy bands, which is not sufficient to estimate the acceleration time at the Sun as we did for Jan14. Thus, we do not have reliable SEP data to test whether the onset times of the SEP and gamma-rays coincide; however, we can compare the height of the CME at the time of the gamma-ray onset with the CME height at SPR found for Jan14. Based on the second-order fit of height as a function of time (from the LASCO CME online catalog), we estimated that the CME was at $\sim 2.5 R_{\odot}$ at the onset time of the Fermi-LAT emission. We found a similar value for Oct13. These heights are significantly smaller than the predicted values reported in Thakur et al. (2014) for the CME heights at SPR as a function of source longitudes from Solar Cycle 23. To test whether these CME heights are compatible with the gamma-ray emission detected from the BTL flares, detailed simulations on particle transport in the solar magnetic field are required. This study is beyond the scope of this paper and will be addressed in a future work.

\subsection{Spatial Distributions}

The RHESSI images show that the 20-50 keV HXRs, due to electron bremsstrahlung, are emitted from $\approx 30^{\prime \prime}\left(L \sim 2 \times 10^{9}\right.$ $\mathrm{cm})$ and $\approx 50^{\prime \prime}\left(L \sim 3.5 \times 10^{9} \mathrm{~cm}\right)$ sources from the tops of loops extending to heights of $\sim 4 \times 10^{9}$ and $\sim 2 \times 10^{10} \mathrm{~cm}$ above the photosphere for the Oct13 and Sep14 flares, respectively. The column depth required for a particle of mass $m$ and kinetic energy $E$ (in units of $m c^{2}$ ), or Lorentz factor $\gamma=E+1=\left(1-\beta^{2}\right)^{-1 / 2}$, to lose all its energy via Coulomb interactions is

$$
\begin{aligned}
N_{\text {Coul }} & =\left(4 \pi r_{0}^{2} \ln \Lambda\right)^{-1}\left(m / m_{e}\right) \int_{0}^{E} \beta^{2} d E \\
& =5 \times 10^{22}\left(m / m_{e}\right) E^{2} / \gamma \mathrm{cm}^{-2}
\end{aligned}
$$

where $r_{0}$ is the classical electron radius and $\ln \Lambda \sim 20$ is the Coulomb logarithm. From Equation (2) we find that the column depth required to stop the $>50 \mathrm{keV}$ electrons that produce the HXRs detected by RHESSI is $\sim 5 \times 10^{20} \mathrm{~cm}^{-2}$. Assuming a density of $n<10^{10} \mathrm{~cm}^{-3}$, the sizes of the loop-top sources imply a column depth of $N_{\mathrm{LT}}<2$ and $3.5 \times 10^{19} \mathrm{~cm}^{-2}$ for the 
two HXR sources, respectively. This means that the energyloss time $\tau_{\text {Coul }} \sim 2 N_{\text {Coul }} /(v n)$ is more than 10 times longer than the crossing time $\tau_{\text {cross }} \equiv L / v=N_{\mathrm{LT}} /(v n)$. These values, together with the results reported in Chen \& Petrosian (2013), suggest that the HXR emission from these two BTL flares are thin-target sources.

If these loop-top sources were to be produced in a thick target, which can come about in two ways, it would be under extreme conditions not likely to be the case here. The first is in the strong diffusion limit, i.e., if the electrons are trapped because the scattering time, $\tau_{\mathrm{sc}}$, is smaller than the crossing time, $\tau_{\text {cross }}$. Note that because $\tau_{\text {Coul }}>\tau_{\text {cross }}$, the scattering cannot be due to Coulomb interactions but it could be due to turbulence. The second possibility is in the weak diffusion limit $\tau_{\text {sc }}>\tau_{\text {cross }}$, where trapping can occur if the field lines converge strongly. In this case we need $\tau_{\mathrm{sc}}>\tau_{\text {Coul }} / \eta^{59}$ (with proportionality constant $\eta$ increasing with increasing field convergence (Petrosian 2016)). These are somewhat extreme conditions requiring either a very high density (therefore increasing the scattering, presumably turbulence) or a somewhat strong field convergence and a "clean" loop with low density and low level of turbulence.

Thus, the HXR emission is most likely due to electron bremsstrahlung from a thin-target loop-top source.

Based on the positions of the Fermi-LAT $>100 \mathrm{MeV}$ emission centroids alone, we cannot exclude that the gammarays come from the loop-top source. In order to investigate this possibility, we rely on Equation (2) to find the column depth required to stop $>350 \mathrm{MeV}$ protons (which produce $>100 \mathrm{MeV}$ photons) and find $N_{\text {Coul }}(350 \mathrm{MeV}) \sim 10^{25} \mathrm{~cm}^{-2}$. This is much larger than the loop-top column depth $N_{\text {LT }} \sim 2-3.5 \times 10^{19} \mathrm{~cm}^{-2}$ so that, in the absence of trapping, we are again dealing with a thin-target loop-top source and a small energy loss compared to what would be the case for emission from a thick-target photospheric source. Consequently, we would need much larger energies of accelerated protons than those given in Table 2 assuming a thick-target scenario. The condition for proton trapping in the loop top (or any coronal trap region, for that matter) is also more extreme than that described above for electrons, and we would need escape times $10^{5}$ times longer than the crossing time. Thus, the emission detected by the Fermi-LAT is most likely due to decay of pions produced by energetic protons interacting in a thick-target photospheric source.

\subsection{Spectral Distributions}

As shown in Lin \& Hudson (1971) and McTiernan \& Petrosian (1990), in a thin-target scenario the relation between the HXR spectral index, $\gamma$, and the number index of the energy spectrum of the emitting electrons ${ }^{60}$ through bremsstrahlung processes, $\delta$, is

$$
\delta= \begin{cases}\gamma-0.5 & \text { non-relativistic case } \\ \gamma & \text { relativistic case }\end{cases}
$$

\footnotetext{
59 According to Malyshkin \& Kulsrud (2001), $\eta \sim \ln \left(B_{0} / B_{L}\right)$, the log of the ratio of magnetic field strengths at the middle and ends of a magnetic bottle.

60 This should not be confused with the electron flux, $F(E)=v N(E)$, index, which in the nonrelativistic limit is $\delta \ln F(E) / d \ln E=\gamma-1.0$, used commonly. In the relativistic regime these indexes are equal and as stated in Equation (3) $\delta=\gamma$.
}

For optically thin synchrotron radiation, the relation between $\delta$ and the microwave photon spectral index, $\alpha$, can be described by the following relations for the relativistic (e.g., Rybicki \& Lightman 1979) and semirelativistic (e.g., Dulk 1985) cases

$$
\delta= \begin{cases}1.1 \alpha+1.36 & \text { semi-relativistic case } \\ 2.0 \alpha+1 & \text { relativistic case }\end{cases}
$$

We will use these relations in the following for the interpretation of the detected emission from the two brightest BTL flares reported in this work. The spectra of the two flares in the $30 \mathrm{keV}-10 \mathrm{MeV}$ energy range are somewhat different so we discuss them separately.

Oct13: As already shown in Pesce-Rollins et al. (2015), both RHESSI and Fermi-GBM detect HXR spectra for the Oct13 flare that are relatively steep (the best-fit value of the power-law photon index $\gamma=3.2 \pm 0.1$ ). However, as shown in Figure 10, there is a tentative indication that the spectrum may harden into a nearly flat part in the $1-10 \mathrm{MeV}$ range, requiring either a similar hardening of the spectrum of the accelerated electrons, as, e.g., described in Park et al. (1997), or a contribution from de-excitation nuclear lines. We found that the inclusion of a $2.223 \mathrm{MeV}$ broad neutron capture line and the nuclear deexcitation lines did not improve the fit significantly and that the best-fit model for the emission observed by Fermi-GBM is a single PL.

As described in Section 5.2, the thin target is most likely, in which case following Equation (3) for the nonrelativistic case $(<100 \mathrm{keV})$ the index of the electron number density $\delta \sim 2.7$. The radio spectrum observed by RSTN, shown in Figure 12, indicates microwave flux of 60 solar flux units $\left(6 \times 10^{5} \mathrm{Jy}\right)$, which peaks at frequency $\nu \sim 5 \mathrm{GHz}$ and declines as $\nu^{-\alpha}$ with the index $\alpha \sim 2$ above this frequency. Based on the relations described in Equation (4), we find that the required power-law index for the number density of electrons to be $\delta \sim 5$ or $\delta \sim 3.5$ in the relativistic or semirelativistic regime, respectively.

These values are steeper than the index $\delta \sim 2.7$ required in the nonrelativistic HXR range, indicating a steepening of the electron spectrum above $1 \mathrm{MeV}$. We therefore conclude that the GBM flux in the energy range $300 \mathrm{keV}-10 \mathrm{MeV}$ is probably not due to the accelerated electrons and no extra component is expected.

Sep14: The best-fit model for the combined HXR FermiGBM gamma-ray emission from the Sep14 flare is a single PL with an exponential decay with photon index $2.06 \pm 0.01$ and a cutoff energy of $90 \pm 7 \mathrm{MeV}$. This is an extremely hard spectrum for a solar flare and if it is produced by a thin-target electron bremsstrahlung model as described above, it would require an electron number spectrum with index $\delta \sim 1.5$ in the nonrelativistic range steepening to an index $\delta \sim 2$ in the relativistic range. However, as noted above, inclusion of the $2.223 \mathrm{MeV}$ line improves the fit slightly, indicating a possible contribution from nuclear de-excitation lines so the steepening of the electron spectrum could be larger.

The radio spectrum of this flare, shown in Figure 12, is also harder. The emission detected by the San Vito station has an index $\alpha \sim 1$ above $1 \mathrm{GHz}$. Again assuming optically thin synchrotron emission would require semirelativistic electrons with electron power-law index $\delta \sim 2.5$, indicating a slightly larger steepening at high energies. If the magnetic field is lower, which could be the case because of the larger loop, the steepening would be even larger $(\delta \sim 3)$. Thus, for the Sep14 
flare a power-law spectrum, steepening at higher energies, is required to explain the HXR and microwave observations from the loop-top region. This, plus a pion-decay component, provides an acceptable fit to the Fermi data from $30 \mathrm{keV}$ to several GeV.

\subsection{Acceleration Site and Mechanism}

The above interpretation of the HXR and MWs indicates that the electrons responsible for these emissions are most likely accelerated in the reconnection region above the loop-top source either by turbulence (Petrosian 2012) or in merging islands manifested in the particle-in-cell simulations (Drake \& Swisdak 2012). The electrons are trapped in the loop-top region long enough (longer than the crossing time of a fraction of second) to produce detectable bremsstrahlung and synchrotron radiation, but most of their energy is lost in thick-target footpoints hidden from near-Earth instruments because of high optical depths (Pesce-Rollins et al. 2015). This scenario can also explain the HXR emission from a BTL flare reported by Krucker et al. (2007). The steepening of the electron spectrum in the semirelativistic regime is due to a decrease in the acceleration rate and not to transport or energy-loss effects.

If the Fermi-LAT emission is from a thick-target photospheric site, then this emission site is located on the visible part of the disk. It cannot come from the occulted AR. The fact that accelerated protons reach the on-disk emission site provides strong evidence that the acceleration site is the CME environment, as suggested by Cliver et al. (1993) and PesceRollins et al. (2015). They would diffuse across a wide range of magnetic fields, some connected to the AR behind the limb and some to the visible side of the Sun. These ions will most likely come from the downstream region of the CME shock while SEPs escape the upstream region. This can account for the differences in their spectral and temporal characteristics. It is then possible that the discrepancy between the spectral characteristics in $1-10 \mathrm{MeV}$ for the GBM and radio observations can be explained by lower-energy $1-100 \mathrm{MeV}$ protons or electrons precipitating to the visible disk side from the downstream region and producing the $1-10 \mathrm{MeV}$ nuclear deexcitation line or bremsstrahlung emission below the chromosphere. These and other possibilities, for example, the production of Fermi-LAT emission by relativistic electrons either in the CME shock, in reconnection regions on current sheets behind the CME, or trapped in a large loop with strong convergence, will be discussed in future papers.

The Fermi-LAT Collaboration acknowledges support from a number of agencies and institutes for both development and the operation of the LAT as well as scientific data analysis. These include NASA and DOE in the United States, CEA/Irfu and IN2P3/CNRS in France, ASI and INFN in Italy, MEXT, KEK, and JAXA in Japan, and the K. A. Wallenberg Foundation, the Swedish Research Council, and the National Space Board in Sweden. Additional support from INAF in Italy and CNES in France for science analysis during the operations phase is also gratefully acknowledged. W.L. was supported by NASA HGI grant NNX16AF78G and LWS grant NNX14AJ49G. V.P., W.L., and F.R.d.C. are supported by NASA grants NNX14AG03G, NNX13AF79G, and NNX12AO70G. V.P. and L.K. are grateful for RFBR grant 15-02-03717. We thank Nariaka Nitta and Meng Jin for friutful discussions on SEPs and magnetic connectivity.

\section{References}

Ackermann, M., Ajello, M., Albert, A., et al. 2014, ApJ, 787, 15 Ackermann, M., Ajello, M., Allafort, A., et al. 2012, ApJ, 745, 144 Ajello, M. A. A., Allafort, A., Baldini, L., et al. 2014, ApJ, 789, 20 Aptekar, R. L., Frederiks, D. D., Golenetskii, S. V., et al. 1995, SSRv, 71, 265 Arnaud, K. A. 1996, in ASP Conf. Ser. 101, Astronomical Data Analysis Software and Systems V, ed. G. H. Jacoby \& J. Barnes (San Francisco, CA: ASP), 17

Atwood, W. B., Abdo, A. A., Ackermann, M., et al. 2009, ApJ, 697, 1071

Barat, C., Trottet, G., Vilmer, N., et al. 1994, ApJL, 425, L109

Chen, Q., \& Petrosian, V. 2013, ApJ, 777, 33

Cliver, E. W., Kahler, S. W., \& Vestrand, W. T. 1993, ICRC, 3, 91

Debrunner, H., Flückiger, E., Grädel, H., Lockwood, J. A., \& McGuire, R. E. 1988, JGRA, 93, 7206

Drake, J. F., \& Swisdak, M. 2012, in APS April Meeting Abstracts, 3-D Particle-in-cell Simulations of the Sawtooth Crash, (Altana, GA), K1.022

Dulk, G. A. 1985, ARA\&A, 23, 169

Fitzpatrick, G., McBreen, S., Connaughton, V., \& Briggs, M. 2012, Proc. SPIE, 8443, 3

Hurford, G. J., Schmahl, E. J., Schwartz, R. A., et al. 2002, SoPh, 210, 61

Krucker, S., White, S. M., \& Lin, R. P. 2007, ApJL, 669, L49

Lin, R. P., \& Hudson, H. S. 1971, SoPh, 17, 412

Malyshkin, L., \& Kulsrud, R. 2001, ApJ, 549, 402

Mattox, J. R., Bertsch, D. L., Chiang, J., et al. 1996, ApJ, 461, 396

McTiernan, J. M., \& Petrosian, V. 1990, ApJ, 359, 541

Meegan, C., Lichti, G., Bhat, P. N., et al. 2009, ApJ, 702, 791

Murphy, R. J., Dermer, C. D., \& Ramaty, R. 1987, ApJS, 63, 721

Murphy, R. J., Kozlovsky, B., Kiener, J., \& Share, G. H. 2009, ApJS, 183, 142

Nakajima, H., Nishio, M., Enome, S., et al. 1994, IEEEP, 82, 705

Nitta, N. V., Aschwanden, M. J., Boerner, P. F., et al. 2013, SoPh, 288, 241

Park, B. T., Petrosian, V., \& Schwartz, R. A. 1997, ApJ, 489, 358

Pesce-Rollins, M., Omodei, N., Petrosian, V., et al. 2015, ApJL, 805, L15

Petrosian, V. 2012, SSRv, 173, 535

Petrosian, V. 2016, ApJ, 830, 28

Rybicki, G. B., \& Lightman, A. P. 1979, Radiative processes in astrophysics (New York: Wiley-Interscience), 393

Saldanha, R., Krucker, S., \& Lin, R. P. 2008, ApJ, 673, 1169

Thakur, N., Gopalswamy, N., Xie, H., et al. 2014, ApJL, 790, L13

Thompson, W. T., \& Wei, K. 2010, SoPh, 261, 215

Vestrand, W. T., \& Forrest, D. J. 1993, ApJL, 409, L69

Vilmer, N., Trottet, G., Barat, C., et al. 1999, A\&A, 342, 575

Wilks, S. S. 1938, Ann. Math. Stat., 9, 60 